\title{
Numerical Simulation of Vertical Random Vibration of Train-Slab Track-Bridge Interaction System by PEM
}

\author{
Zhi-ping Zeng, ${ }^{1,2,3}$ Zhi-wu Yu, ${ }^{1,3}$ Yan-gang Zhao, ${ }^{1,2}$ Wen-tao Xu, \\ Ling-kun Chen, ${ }^{3,5}$ and Ping Lou ${ }^{1,3}$ \\ ${ }^{1}$ School of Civil Engineering, Railway Campus, Central South University, Changsha 410075, China \\ ${ }^{2}$ Department of Architecture, Kanagawa University, Yokohama 221-8686, Japan \\ ${ }^{3}$ National Engineering Laboratory for High-Speed Railway Construction, Central South University, Changsha 410075, China \\ ${ }^{4}$ School of Mechanics and Engineering Science, Zhengzhou University, Zhengzhou 450001, China \\ ${ }^{5}$ College of Civil Science and Engineering, Yangzhou University, Yangzhou 225127, China
}

Correspondence should be addressed to Zhi-ping Zeng; hzzp7475@126.com

Received 2 March 2014; Revised 12 June 2014; Accepted 13 June 2014; Published 12 August 2014

Academic Editor: Ahmet S. Yigit

Copyright (C) 2014 Zhi-ping Zeng et al. This is an open access article distributed under the Creative Commons Attribution License, which permits unrestricted use, distribution, and reproduction in any medium, provided the original work is properly cited.

\begin{abstract}
The paper describes the numerical simulation of the vertical random vibration of train-slab track-bridge interaction system by means of finite element method and pseudoexcitation method. Each vehicle is modeled as four-wheelset mass-spring-damper system with two-layer suspension systems. The rail, slab, and bridge girder are modeled by three-layer elastic Bernoulli-Euler beams connected with each other by spring and damper elements. The equations of motion for the entire system are derived according to energy principle. By regarding rail irregularity as a series of multipoint, different-phase random excitations, the random load vectors of the equations of motion are obtained by pseudoexcitation method. Taking a nine-span simply supported beam bridge traveled by a train consisting of 8 vehicles as an example, the vertical random vibration responses of the system are investigated. Firstly, the suitable number of discrete frequencies of rail irregularity is obtained by numerical experimentations. Secondly, the reliability and efficiency of pseudoexcitation method are verified through comparison with Monte Carlo method. Thirdly, the random vibration characteristics of train-slab track-bridge interaction system are analyzed by pseudoexcitation method. Finally, applying the $3 \sigma$ rule for Gaussian stochastic process, the maximum responses of train-slab track-bridge interaction system with respect to various train speeds are studied.
\end{abstract}

\section{Introduction}

The dynamic analysis of railway track or bridge subjected to a moving train has long been a subject of great interest in the field of civil engineering [1-10]. As is well known [11-19], rail irregularity, with random nature, is one of the most important factors that can amplify the vibration responses of train-track/bridge interaction system. The dynamic responses in most of the previous researches are usually calculated by using only one time-history sample of rail irregularity. From the point of view of random vibration, those results can merely represent one of a series of possible random samples due to the fact that each time-history sample generated from the PSD function of rail irregularity is random.
With the increasing speed and axle load of train, the random vibration of train-track/bridge interaction system has received more and more attention and is usually analyzed by means of Monte Carlo method (MCM) [12, 16]. In this approach, the time-history samples of dynamic responses are calculated by using a series of samples of rail irregularity firstly, and then the statistical characteristics of responses are obtained according to the maximum values of each sample of dynamic responses. In order to ensure the reliability of the simulation, tens, hundreds, or even thousands of samples must be calculated, which may result in intolerable computer time, especially for the sophisticated train-slab track-bridge interaction system.

Pseudoexcitation method (PEM), originally developed from the field of bridge seismic resistance [20], has been 
applied to analyze efficiently and accurately the linear timeindependent or time-dependent systems with stationary or nonstationary random excitations [21-25]. Lu et al. [23] studied the stationary random vibration responses of vehicleballasted track interaction system by PEM in which a timeindependent vehicle-track interaction model was adopted. By adopting a train-bridge interaction element with the track structure omitted [26], PEM was applied to research the nonstationary random vibration of time-dependent trainbridge interaction system [24].

With the increasing use of ballastless track on bridge in modern high-speed railway, this paper investigates the vertical random vibration responses of slab ballastless track and bridge subjected to a moving train based on finite element method (FEM) and PEM. The train, slab track, and bridge are regarded as an integrated system. Each moving vehicle is modeled as four-wheelset mass-spring-damper system with two-layer suspension systems possessing 6 degrees of freedom (DOFs) [17]. The rail, slab, and bridge girder are modeled by three-layer elastic Bernoulli-Euler beams [17, 19, 27]. The elasticity and damping properties of the fastener and bridge bearing are represented by discrete springs and dampers. Simultaneously, the elasticity and damping properties of the concrete asphalt layer (CA layer) are represented by continuous springs and dampers $[27,28]$. The equations of motion of finite element form for the entire system are derived by means of energy principle [28]. The effects of rail irregularity are regarded as a series of multipoint, differentphase random excitations by taking time lags between the wheels into account. The random excitations between rail and wheels caused by rail irregularity are then transformed into a series of deterministic pseudoharmonic excitation vectors by PEM, so that the random vibration responses of train, slab track, and bridge can be obtained by step-bystep integration method such as Newmark- $\beta$ method [29] or Wilson- $\theta$ method [30]. Taking a nine-span simply supported beam bridge traveled by a train consisting of 2 motor cars and 6 trailer cars as an example, the suitable number of discrete frequencies of rail irregularity for calculating the random vibration responses of the system is obtained by numerical experimentations, the reliability and efficiency of the random vibration responses obtained by PEM are verified through comparison with those by MCM; the random vibration characteristics of train-slab track-bridge interaction system are analyzed by PEM, and finally the maximum responses of train-slab track-bridge interaction system with respect to various train moving speeds are studied by applying the $3 \sigma$ rule for Gaussian stochastic process.

\section{Models of Train, Slab Track, and Bridge}

2.1. Model of Train. Figure 1 shows a train consisting of a series of four-wheelset vehicles moving with constant speed $v$ on a slab track structure resting on a series of simply supported beams to model a railway bridge and the two approach subgrades. The train consists of the front and rear motor cars numbered 1 and 2 , respectively, and $N_{v}$ trailer cars numbered $1,2, \ldots, N_{v}$ from right to left. It is assumed that the downward vertical displacements and clockwise direction rotation of train are taken as positive and that they are measured with reference to their respective static equilibrium positions before moving upon the track concerned.

Each trailer car in the train is modeled as a mass-springdamper system consisting of one carbody, two bogies, four wheelsets, and two-stage suspensions. As shown in Figure 1, the carbody rests on the front and rear bogies, each of which in turn is supported by two wheelsets. The carbody is modeled as a rigid body with a mass $m_{c}$ and a moment of inertia $J_{c}$ about the transverse horizontal axis through its center of gravity. Similarly, each bogie is considered as a rigid body having a mass $m_{t}$ and a moment of inertia $J_{t}$ about the transverse horizontal axis through its center of gravity. Each wheelset has a mass $m_{w}$. The spring and shock absorber in the primary suspension for each wheelset are characterized by spring stiffness $k_{p}$ and damping coefficient $c_{p}$, respectively. Likewise, the secondary suspension between carbody and each bogie is characterized by spring stiffness $k_{s}$ and damping coefficient $c_{s}$. As the carbody is assumed to be rigid, the motions of the $j$ th trailer carbody may be described by the vertical displacement $y_{c j}$ and rotation $\theta_{c j}$ at its center of gravity, where the subscript $j$ denotes the trailer car number. Similarly, the motions of the front bogie of the $j$ th trailer car may be described by the vertical displacement $y_{t 1 j}$ and rotation $\theta_{t 1 j}$ at its center of gravity; the motions of the rear bogie of the $j$ th trailer car may be described by the vertical displacement $y_{t 2 j}$ and rotation $\theta_{t 2 j}$ at its center of gravity. The motions of the four wheelsets from right to left of the $j$ th trailer car may be described by the vertical displacements $y_{w 1 j}, y_{w 2 j}, y_{w 3 j}$, and $y_{w 4 j}$, respectively. Therefore, the total number of DOFs for each trailer car is ten. However, it is assumed that each wheelset of all vehicles is always in full contact with the rail in this paper; that is, the vertical displacement of each wheelset is constrained by the displacement of the rail. Consequently, the independent DOFs for each trailer car become six.

Each motor car in the train is also modeled as a massspring-damper system consisting of one carbody, two bogies, four wheelsets, and two-stage suspensions. The carbody has a mass $m_{J c}$ and a moment of inertia $J_{J c}$. Each bogie has a mass $m_{J t}$ and a moment of inertia $J_{J t}$. Each wheelset has a mass $m_{J w}$. The primary suspension is characterized by spring stiffness $k_{J p}$ and damping coefficient $c_{J p}$. Likewise, the secondary suspension is characterized by spring stiffness $k_{J s}$ and damping coefficient $c_{J s}$. The independent DOFs for each motor car include, too, the carbody vertical displacement $y_{J c i}$ and rotation $\theta_{J c i}$, the front bogie vertical displacement $y_{J t 1 i}$ and rotation $\theta_{J t 1 i}$, and the rear bogie vertical displacement $y_{J t 2 i}$ and rotation $\theta_{J t 2 i}$, where the subscript $i$ denotes the motor car number.

2.2. Models of Slab Track and Bridge. As shown in Figure 1, the rail, slab, and bridge girder are all modeled as elastic Bernoulli-Euler beam. The two rails are effectively treated as one in the subsequent analysis. On the basis of FEM, the rail, slab, and bridge girder are all divided into a series of beam elements of equal length $l$. The elasticity and damping properties 


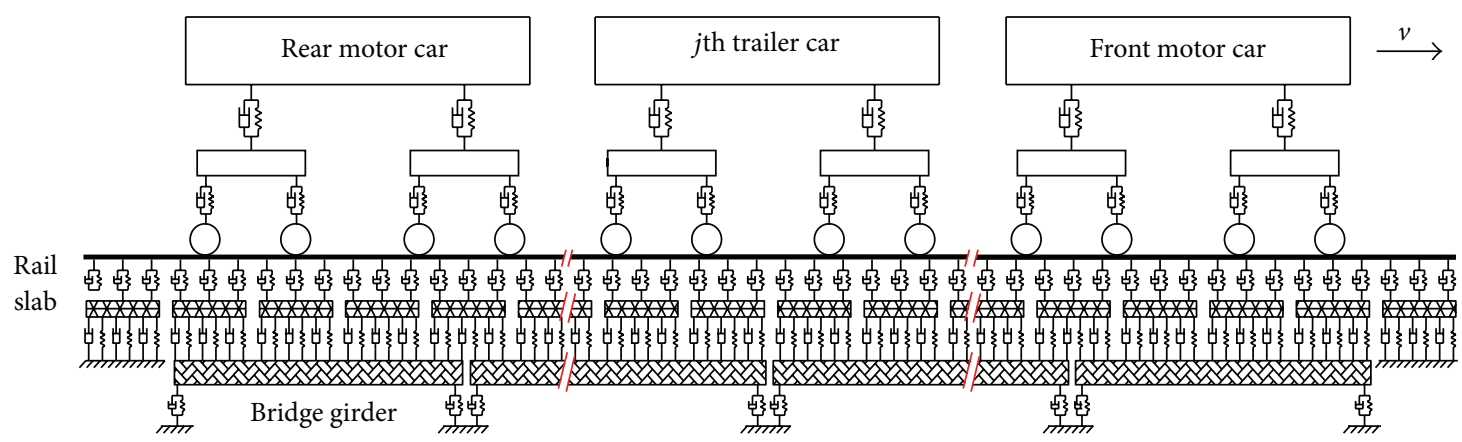

(a)

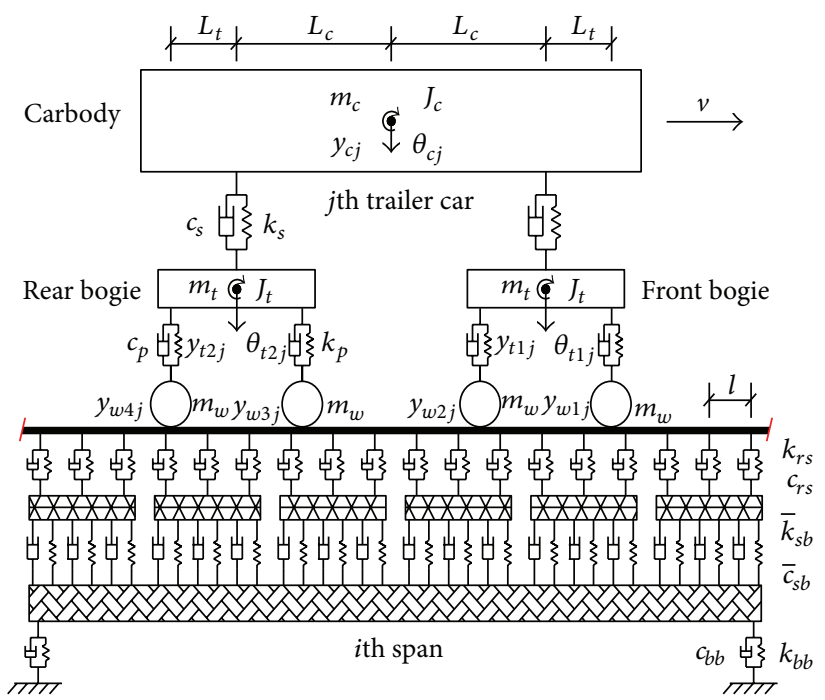

(b)

FIGURE 1: Model of train-slab track-bridge interaction system. (a) A train moving upon a slab track resting on a multispan simply supported beam bridge. (b) The $j$ th trailer car moving upon the $i$ th span simply supported beam.

of the fastener are represented by discrete massless springs with stiffness $k_{r s}$ and dampers with damping coefficient $c_{r s}$. The elasticity and damping properties of the CA layer underlying the slab are represented by continuous massless springs with stiffness $\bar{k}_{s b}$ and dampers with damping coefficient $\bar{c}_{s b}$. Simultaneously, the elasticity and damping properties of the bridge bearing are represented by massless springs with stiffness $k_{b b}$ and dampers with damping coefficient $c_{b b}$. It is assumed that the damping of the rail and slab is neglected $[19,31]$, and the bridge girder has linear viscous damping $[15,32]$. In addition, by neglecting axial deformations of the rail, slab, and bridge girder, each node of the rail, slab, and bridge girder has two DOFs, that is, vertical displacement and rotation about an axis normal to the plane of paper. The cubic Hermitian interpolation polynomials are used as shape functions of the rail, slab, and bridge girder elements.

It is assumed also that the downward deflections of rail, slab, and bridge are taken as positive and that they are measured with reference to their respective vertical static equilibrium positions. Let $r(x)$ denote the initial vertical irregularities of rail and be measured with reference to smooth profile of rail; that is, $r(x)=0$, if the top surface of rail is smooth. Likewise, it is considered positive in the downward direction.

\section{Equations of Motion for a Train-Slab Track- Bridge Interaction System}

By using the energy principle, such as the principle of a stationary value of total potential energy of a dynamic system [28], one can derive the equations of motion written in submatrix for the train-slab track-bridge interaction system as

$$
\begin{gathered}
{\left[\begin{array}{cccc}
\mathbf{M}_{t t} & 0 & 0 & 0 \\
0 & \mathbf{M}_{r r} & 0 & 0 \\
0 & 0 & \mathbf{M}_{s s} & 0 \\
0 & 0 & 0 & \mathbf{M}_{b b}
\end{array}\right]\left\{\begin{array}{l}
\ddot{\mathbf{X}}_{t} \\
\ddot{\mathbf{X}}_{r} \\
\ddot{\mathbf{X}}_{s} \\
\ddot{\mathbf{X}}_{b}
\end{array}\right\}} \\
+\left[\begin{array}{cccc}
\mathbf{C}_{t t} & \mathbf{C}_{t r} & 0 & 0 \\
\mathbf{C}_{r t} & \mathbf{C}_{r r} & \mathbf{C}_{s r} & 0 \\
0 & \mathbf{C}_{r s} & \mathbf{C}_{s s} & \mathbf{C}_{s b} \\
0 & 0 & \mathbf{C}_{b s} & \mathbf{C}_{b b}
\end{array}\right]\left\{\begin{array}{l}
\dot{\mathbf{X}}_{t} \\
\mathbf{X}_{r} \\
\dot{\mathbf{X}}_{s} \\
\dot{\mathbf{X}}_{b}
\end{array}\right\} \\
+\left[\begin{array}{cccc}
\mathbf{K}_{t t} & \mathbf{K}_{t r} & 0 & 0 \\
\mathbf{K}_{r t} & \mathbf{K}_{r r} & \mathbf{K}_{r s} & 0 \\
0 & \mathbf{K}_{s r} & \mathbf{K}_{s s} & \mathbf{K}_{s b} \\
0 & 0 & \mathbf{K}_{b s} & \mathbf{K}_{b b}
\end{array}\right]\left\{\begin{array}{l}
\mathbf{X}_{t} \\
\mathbf{X}_{r} \\
\mathbf{X}_{s} \\
\mathbf{X}_{b}
\end{array}\right\}=\left\{\begin{array}{l}
\mathbf{F}_{t} \\
\mathbf{F}_{r} \\
\mathbf{F}_{s} \\
\mathbf{F}_{b}
\end{array}\right\},
\end{gathered}
$$


where the subscripts " $t$," " $r$," "s," and " $b$ " denote the train, rail, slab, and bridge girder, respectively. The displacement vector, the matrices of mass, stiffness, and damping, as well as the load vector for the train, rail, slab, and bridge girder, are explained as follows.

3.1. Displacement Vectors. The total train displacement vector $\mathbf{X}_{t}$ with order $T_{\text {dof }}\left(T_{\text {dof }}=\left(6 \times N_{v}\right)+6 \times 2\right)$ can be written as

$$
\mathbf{X}_{t}=\left[\begin{array}{llllll}
\mathbf{X}_{J 1} & \mathbf{X}_{v 1} & \mathbf{X}_{v 2} & \cdots & \mathbf{X}_{v N_{v}} & \mathbf{X}_{J 2}
\end{array}\right]^{\mathbf{T}}
$$

where the superscript "T" denotes the transpose of the vector and $\mathbf{X}_{v j}\left(j=1,2, \ldots, N_{v}\right)$ and $\mathbf{X}_{J i}(i=1,2)$ are the displacement vectors of the $j$ th trailer car and the $i$ th motor car, respectively, which can be expressed as

$$
\begin{gathered}
\mathbf{X}_{v j}=\left[\begin{array}{llllll}
y_{c j} & \theta_{v j} & y_{t 1 j} & \theta_{t 1 j} & y_{t 2 j} & \theta_{t 2 j}
\end{array}\right], \\
\mathbf{X}_{J i}=\left[\begin{array}{llllll}
y_{J c i} & \theta_{J c i} & y_{J t 1 i} & \theta_{J t 1 i} & y_{J t 2 i} & \theta_{J t 2 i}
\end{array}\right] .
\end{gathered}
$$

The displacement vector of rail $\mathbf{X}_{r}$ with order $N_{r} \times 1$ can be written as

$$
\mathbf{X}_{r}=\left[\begin{array}{llll}
q_{r 1} & q_{r 2} & \cdots & q_{r N_{r}}
\end{array}\right]^{\mathrm{T}},
$$

where $N_{r}$ denotes the total number of DOFs of rail.

The displacement vector $\mathbf{X}_{s}$ with order $\bar{N}_{s} \times 1$ for a series of continuously supported beams to model slab can be written as

$$
\mathbf{X}_{s}=\left[\begin{array}{llll}
\mathbf{X}_{s 1} & \mathbf{X}_{s 2} & \cdots & \mathbf{X}_{s N_{s}}
\end{array}\right]^{\mathrm{T}},
$$

where $\mathbf{X}_{s i}\left(i=1,2, \ldots, N_{s}\right)$ denotes the displacement vector of the $i$ th slab, $N_{s}$ denotes the total number of slab, and $\bar{N}_{s}$ denotes the total number of DOFs of all slab. $\mathbf{X}_{s i}$ with order $1 \times \bar{n}_{s i}$ and $\bar{N}_{s}$ can be expressed as

$$
\begin{gathered}
\mathbf{X}_{s i}=\left[\begin{array}{cccc}
q_{s 1} & q_{s 2} & \cdots & q_{s \bar{n}_{s i}}
\end{array}\right], \\
\bar{N}_{s}=\sum_{i=1}^{N_{s}} \bar{n}_{s i} .
\end{gathered}
$$

The displacement vector $\mathbf{X}_{b}$ with order $\bar{N}_{b} \times 1$ for a series of simply supported beams to model the bridge can be written as

$$
\mathbf{X}_{b}=\left[\begin{array}{llll}
\mathbf{X}_{b 1} & \mathbf{X}_{b 2} & \cdots & \mathbf{X}_{b N_{b}}
\end{array}\right]^{\mathrm{T}}
$$

where $\mathbf{X}_{b i}\left(i=1,2, \ldots, N_{b}\right)$ denotes the displacement vector of the $i$ th bridge girder, $N_{b}$ denotes the total number of bridge girder, and $\bar{N}_{b}$ denotes the total number of DOFs of all bridge girder. $\mathbf{X}_{b i}$ with order $1 \times \bar{n}_{b i}$ and $\bar{N}_{b}$ can be expressed as

$$
\begin{gathered}
\mathbf{X}_{b i}=\left[\begin{array}{cccc}
q_{b 1} & q_{b 2} & \cdots & q_{s \bar{n}_{b i}}
\end{array}\right], \\
\bar{N}_{b}=\sum_{i=1}^{N_{b}} \bar{n}_{b i} .
\end{gathered}
$$

3.2. Matrices for Train. The matrices of train are marked with the subscript " $t t$." The mass matrix $\mathbf{M}_{t t}$ of train, with order $\left(6 \times N_{v}+6 \times 2\right) \times\left(6 \times N_{v}+6 \times 2\right)$, can be written as

$$
\mathbf{M}_{t t}=\operatorname{diag}\left[\begin{array}{llllll}
\mathbf{M}_{J 1} & \mathbf{M}_{v 1} & \mathbf{M}_{v 2} & \cdots & \mathbf{M}_{v N_{v}} & \mathbf{M}_{J 2}
\end{array}\right]
$$

where $\mathbf{M}_{v j}$ and $\mathbf{M}_{J i}$ with order $6 \times 6$ denote the mass matrices of the $j$ th trailer car and $i$ th motor car, respectively, and can be expressed as

$$
\begin{gathered}
\mathbf{M}_{v j}=\operatorname{diag}\left[\begin{array}{llllll}
m_{c} & J_{c} & m_{t} & J_{t} & m_{t} & J_{t}
\end{array}\right], \\
\mathbf{M}_{J i}=\operatorname{diag}\left[\begin{array}{lllllll}
m_{J c} & J_{J c} & m_{J t} & J_{J t} & m_{J t} & J_{J t}
\end{array}\right] .
\end{gathered}
$$

The stiffness matrix $\mathbf{K}_{t t}$ of train, with order $\left(6 \times N_{v}+6 \times 2\right) \times$ $\left(6 \times N_{v}+6 \times 2\right)$, can be written as

$$
\mathbf{K}_{t t}=\operatorname{diag}\left[\begin{array}{llllll}
\mathbf{K}_{J 1} & \mathbf{K}_{v 1} & \mathbf{K}_{v 2} & \cdots & \mathbf{K}_{v N_{v}} & \mathbf{K}_{J 2}
\end{array}\right],
$$

where $\mathbf{K}_{v j}$ and $\mathbf{K}_{J i}$ with order $6 \times 6$, denoting the stiffness matrices of the $j$ th trailer car and $i$ th motor car, respectively, can be expressed as

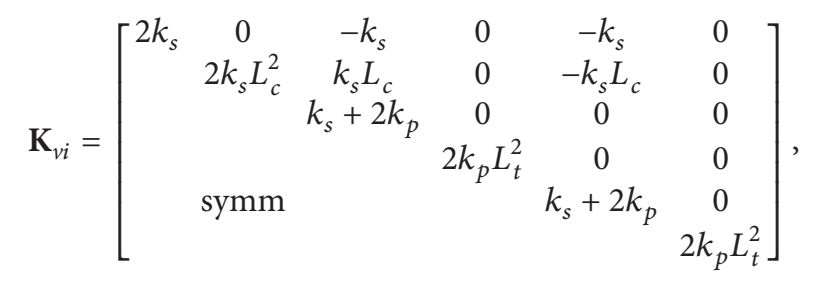

$\mathbf{K}_{J i}$

$$
=\left[\begin{array}{cccccc}
2 k_{J s} & 0 & -k_{J s} & 0 & -k_{J s} & 0 \\
& 2 k_{J s} L_{J c}^{2} & k_{J s} L_{J c} & 0 & -k_{J s} L_{J c} & 0 \\
& & k_{J s}+2 k_{J p} & 0 & 0 & 0 \\
& & & 2 k_{J p} L_{J t}^{2} & 0 & 0 \\
& & & & & \\
& & & & & \\
& & & & & 2 k_{J s}+2 k_{J p} L_{J t}^{2}
\end{array}\right]
$$

in which $L_{c}$ denotes half of longitudinal distance between the centers of gravity of trailer car's front and rear bogies, $L_{J c}$ denotes half of longitudinal distance between the centers of gravity of motor car's front and rear bogies, $L_{t}$ denotes half of trailer car's bogie axle base, and $L_{J t}$ denotes half of motor car's bogie axle base.

The damping matrix $\mathbf{C}_{t t}$ of train with order $\left(6 \times N_{v}+6 \times 2\right) \times$ $\left(6 \times N_{v}+6 \times 2\right)$ can be obtained by simply replacing $k$ in the corresponding stiffness matrix $\mathbf{K}_{t t}$ by $c$.

3.3. Matrices for Rail, Slab, and Bridge. The matrices of rail are marked with the subscript " $r r$." The mass matrix $\mathbf{M}_{r r}$ of rail, with order $N_{r} \times N_{r}$, can be written as

$$
\mathbf{M}_{r r}=\mathbf{M}_{r r 1}+\mathbf{M}_{r r 2}+\mathbf{M}_{r r 3}
$$


with

$$
\begin{aligned}
& \mathbf{M}_{r r 1}=\sum_{g=1}^{n_{r}} \int_{0}^{l} \bar{m}_{r} \mathbf{N}_{r, e g}^{\mathbf{T}} \mathbf{N}_{r, e g} d \xi, \\
& \mathbf{M}_{r r 2}=\sum_{j=1}^{N_{v}} \sum_{h=1}^{4} m_{w} \mathbf{N}_{j h}^{\mathbf{T}} \mathbf{N}_{j h} \\
& \mathbf{M}_{r r 3}=\sum_{i=1}^{2} \sum_{h=1}^{4} m_{J w} \mathbf{N}_{J i h}^{\mathrm{T}} \mathbf{N}_{J i h}, \\
& \mathbf{N}_{r, e g}=\left[\begin{array}{llllllllllll}
0 & 0 & \cdots & 0 & N_{r 1} & N_{r 2} & N_{r 3} & N_{r 4} & 0 & \cdots & 0 & 0
\end{array}\right], \\
& \mathbf{N}_{j h}=\left[\begin{array}{llllllllllll}
0 & 0 & \cdots & 0 & N_{r 1} & N_{r 2} & N_{r 3} & N_{r 4} & 0 & \cdots & 0 & 0
\end{array}\right]_{\xi=\xi_{j h}}, \\
& \mathbf{N}_{\text {rih }}=\left[\begin{array}{lllllllllllll}
0 & 0 & \cdots & 0 & N_{r 1} & N_{r 2} & N_{r 3} & N_{r 4} & 0 & \cdots & 0 & 0
\end{array}\right]_{\xi=\xi_{\text {ilh }}}, \\
& N_{r 1}=1-3\left(\frac{\xi}{l}\right)^{2}+2\left(\frac{\xi}{l}\right)^{3} \text {, } \\
& N_{r 2}=\xi\left[1-2\left(\frac{\xi}{l}\right)+\left(\frac{\xi}{l}\right)^{2}\right] \text {, } \\
& N_{r 3}=3\left(\frac{\xi}{l}\right)^{2}-2\left(\frac{\xi}{l}\right)^{3} \\
& N_{r 4}=\xi\left[\left(\frac{\xi}{l}\right)^{2}-\left(\frac{\xi}{l}\right)\right] \text {, }
\end{aligned}
$$

where $\mathbf{M}_{r r 1}$, with order $N_{r} \times N_{r}$, represents the overall mass matrix of the rail itself, $\bar{m}_{r}$ represents rail mass per unit length, $n_{r}$ represents the total number of rail elements, $\xi$ represents the local coordinate measured from the left node of a beam element, and $\mathbf{N}_{r, e g}$, with order $1 \times N_{r}$, represents the shape function matrix for the $g$ th rail element. It should be noted that each element is zero in $\mathbf{N}_{r, e g}$ except for those corresponding to the four DOFs of the two nodes of the $g$ th rail element. $\mathbf{M}_{r r 2}$, with order $N_{r} \times N_{r}$, represents the overall mass matrix induced by all the wheel masses of trailer cars; $\mathbf{N}_{j h}$, with order $1 \times N_{r}$, represents the shape function matrix for the rail element, evaluated at the position of the $h$ th wheelset of the $j$ th trailer car $; \xi_{j 1}, \xi_{j 2}, \xi_{j 3}$, and $\xi_{j 4}$, respectively, represent the distances between the 1st, 2nd, 3rd, and 4th wheelsets of the $j$ th trailer car and the left node of the rail element upon which the wheelset is acting. It should be noted that each element is zero in $\mathbf{N}_{j h}$ except those corresponding to the four DOFs of the two nodes of the rail element upon which the $h$ th wheelset of the $j$ th trailer car is acting. $\mathbf{N}_{j h}$ is time dependent as the $h$ th wheelset of the $j$ th trailer car moves from one position to another within one rail element. As the $h$ th wheelset of the $j$ th trailer car moves to the next rail element, $\mathbf{N}_{j h}$ will shift in position corresponding to the DOFs of the rail element where the $h$ th wheelset of the $j$ th trailer car is positioned. Similarly, $\mathbf{M}_{r r 3}$, with order $N_{r} \times N_{r}$, represents the overall mass matrix induced by all the wheel masses of motor cars; $\mathbf{N}_{i i h}$, with order $1 \times N_{r}$, represents the shape function matrix for the rail element, evaluated at the position of the $h$ th wheelset of the $i$ th motor car; $\xi_{J i 1}, \xi_{J i 2}, \xi_{J i 3}$, and $\xi_{J i 4}$, respectively, represent the distances between each wheelset of the $i$ th motor car and the left node of the corresponding rail element upon which the wheelset is acting. It should be noted too for $\mathbf{N}_{J i h}$ that, apart from those elements corresponding to the four DOFs of the two nodes of the rail element upon which the $h$ th wheelset of the $i$ th motor car is acting, all other elements are zero. $\mathbf{N}_{J i h}$ is also time dependent.

The stiffness matrix $\mathbf{K}_{r r}$ of rail, with order $N_{r} \times N_{r}$, can be similarly expressed in terms of the overall stiffness matrix $\mathbf{K}_{r r 1}$ of the rail itself, overall stiffness matrix $\mathbf{K}_{r r 2}$ induced by all trailer cars, overall stiffness matrix $\mathbf{K}_{r r 3}$ induced by all motor cars, and overall stiffness matrix $\mathbf{K}_{r r 4}$ induced by the stiffness of all fasteners as

$$
\mathbf{K}_{r r}=\mathbf{K}_{r r 1}+\mathbf{K}_{r r 2}+\mathbf{K}_{r r 3}+\mathbf{K}_{r r 4}
$$

with

$$
\begin{aligned}
& \mathbf{K}_{r r 1}=\sum_{g=1}^{n_{r}} \int_{0}^{l} E_{r} I_{r} \mathbf{N}_{r, e g}^{\prime \prime T} \mathbf{N}_{r, e g}^{\prime \prime} d \xi, \\
& \mathbf{K}_{r r 2}=\sum_{j=1}^{N_{v}} \sum_{h=1}^{4} k_{p} \mathbf{N}_{j h}^{\mathrm{T}} \mathbf{N}_{j h}, \\
& \mathbf{K}_{r r 3}=\sum_{i=1}^{2} \sum_{h=1}^{4} k_{J p} \mathbf{N}_{J i h}^{\mathrm{T}} \mathbf{N}_{J i h}, \\
& \mathbf{K}_{r r 4}=\sum_{p=1}^{n_{p}} k_{r s} \mathbf{N}_{r, p}^{\mathrm{T}} \mathbf{N}_{r, p} \\
& \mathbf{N}_{r, p}=\left[\begin{array}{llllllllllll}
0 & 0 & \cdots & 0 & N_{r 1} & N_{r 2} & N_{r 3} & N_{r 4} & 0 & \cdots & 0 & 0
\end{array}\right]_{\xi=\xi_{r, p}},
\end{aligned}
$$

where $E_{r}$ denotes Young's modulus of the rail and $I_{r}$ denotes the constant moment of inertia of the rail cross section and the prime differentiation with respect to local coordinate $\xi$. In the formulation of $\mathbf{K}_{r r 4}$, the shape function matrix $\mathbf{N}_{r, p}(p=$ $\left.1,2, \ldots, n_{p}\right)$ of order $1 \times N_{r}$ for the rail element is evaluated at the position of the $p$ th fastener; $\xi_{r, p}$ denotes the distance between the $p$ th fastener and the left node of the rail element containing the $p$ th fastener, and $n_{p}$ denotes the total number of fastener. It should be noted for $\mathbf{N}_{r, p}$ that, apart from those elements corresponding to four DOFs of the two nodes of the rail element containing the $p$ th fastener, all other elements are zero.

With omission of the damping of the rail itself, the damping matrix $\mathbf{C}_{r r}$ of rail, with order $N_{r} \times N_{r}$, can be written in terms of the overall damping matrix $\mathbf{C}_{r r 2}$ induced by all trailer cars, overall damping matrix $\mathbf{C}_{r r 3}$ induced by all motor cars, and overall damping matrix $\mathbf{C}_{r r 4}$ induced by the damping of all fasteners as

$$
\mathbf{C}_{r r}=\mathbf{C}_{r r 2}+\mathbf{C}_{r r 3}+\mathbf{C}_{r r 4}
$$

with

$$
\begin{aligned}
\mathbf{C}_{r r 2} & =\sum_{j=1}^{N_{v}} \sum_{h=1}^{4} c_{p} \mathbf{N}_{j h}^{\mathrm{T}} \mathbf{N}_{j h}, \\
\mathbf{C}_{r r 3} & =\sum_{i=1}^{2} \sum_{h=1}^{4} c_{J p} \mathbf{N}_{J i h}^{\mathrm{T}} \mathbf{N}_{j h}, \\
\mathbf{C}_{r r 4} & =\sum_{p=1}^{n_{p}} c_{r s} \mathbf{N}_{r, p}^{\mathrm{T}} \mathbf{N}_{r, p} .
\end{aligned}
$$


The matrices of slab, with order $\bar{N}_{s} \times \bar{N}_{s}$, are marked with the subscript "ss" and can be written in terms of the matrices of the slab itself, the matrices induced by fastener on the slab, and the matrices induced by CA layer underlying the slab. The matrices for bridge, with order $\bar{N}_{b} \times \bar{N}_{b}$, are marked with the subscript " $b b$ " and can be written in terms of the matrices of the bridge girder itself, the matrices induced by CA layer lying on the bridge girder, and the matrices induced by bearing at the support of bridge girder. The matrices of slab and bridge are not derived in detail here but can be obtained by following the similar procedure for derivation of rail matrices.

3.4. Matrices for Train-Rail-Slab-Bridge Interaction. The matrices for train-rail interaction are marked with subscript "tr" or " $r t$." The stiffness matrices $\mathbf{K}_{t r}$ of order $T_{\text {dof }} \times N_{r}, \mathbf{K}_{r t}$ of order $N_{r} \times T_{\text {dof }}, \mathbf{C}_{t r}$ of order $T_{\text {dof }} \times N_{r}$, and $\mathbf{C}_{r t}$ of order $N_{r} \times T_{\text {dof }}$ induced by the interaction between the train and rail can be written, respectively, as

$$
\begin{gathered}
\mathbf{K}_{t r}=\sum_{j=1}^{N_{v}} \sum_{h=1}^{4} \mathbf{K}_{v_{j}-r_{h}}+\sum_{i=1}^{2} \sum_{h=1}^{4} \mathbf{K}_{J_{i}-r_{h}}, \\
\mathbf{K}_{r t}=\mathbf{K}_{t r}^{\mathbf{T}}, \\
\mathbf{C}_{t r}=\sum_{j=1}^{N_{v}} \sum_{h=1}^{4} \mathbf{C}_{v_{j}-r_{h}}+\sum_{i=1}^{2} \sum_{h=1}^{4} \mathbf{C}_{J_{i}-r_{h}}, \\
\mathbf{C}_{r t}=\mathbf{C}_{t r}^{\mathrm{T}},
\end{gathered}
$$

with

$$
\begin{aligned}
\mathbf{K}_{v_{j}-r_{1}}=\left[\begin{array}{c}
0 \\
0 \\
\vdots \\
-k_{p} \mathbf{N}_{j 1} \\
-k_{p} L_{t} \mathbf{N}_{j 1} \\
\vdots \\
0 \\
0
\end{array}\right]_{T_{\mathrm{dof}} \times N_{r}}, \quad \mathbf{K}_{v_{j}-r_{2}}=\left[\begin{array}{c}
0 \\
0 \\
\vdots \\
-k_{p} \mathbf{N}_{j 2} \\
k_{p} L_{t} \mathbf{N}_{j 2} \\
\vdots \\
0 \\
0
\end{array}\right]_{T_{\mathrm{dof}} \times N_{r}} \\
\mathbf{K}_{v_{j}-r_{3}}=\left[\begin{array}{c} 
\\
0 \\
0 \\
-k_{p} \mathbf{N}_{j 3} \\
-k_{p} L_{t} \mathbf{N}_{j 3} \\
\vdots \\
0 \\
0
\end{array}\right]_{T_{\mathrm{dof}} \times N_{r}}, \mathbf{K}_{v_{j}-r_{4}}=\left[\begin{array}{c} 
\\
\vdots \\
-k_{p} \mathbf{N}_{j 4} \\
k_{p} L_{t} \mathbf{N}_{j 4} \\
\vdots \\
0 \\
0
\end{array}\right]_{T_{\mathrm{dof}} \times N_{r}},
\end{aligned}
$$

$$
\begin{aligned}
\mathbf{C}_{v_{j}-r_{1}}= & {\left[\begin{array}{c}
0 \\
0 \\
\vdots \\
-c_{p} \mathbf{N}_{j 1} \\
-c_{p} L_{t} \mathbf{N}_{j 1} \\
\vdots \\
0 \\
0
\end{array}\right]_{T_{\mathrm{dof}} \times N_{r}}, \quad \mathbf{C}_{v_{j}-r_{2}}=\left[\begin{array}{c}
0 \\
0 \\
\vdots \\
-c_{p} \mathbf{N}_{j 2} \\
c_{p} L_{t} \mathbf{N}_{j 2} \\
\vdots \\
0 \\
0
\end{array}\right]_{T_{\mathrm{dof}} \times N_{r}}, } \\
\mathbf{C}_{v_{j}-r_{3}}= & {\left[\begin{array}{c}
0 \\
0 \\
\vdots \\
-c_{p} \mathbf{N}_{j 3} \\
-c_{p} L_{t} \mathbf{N}_{j 3} \\
\vdots \\
0 \\
0
\end{array}\right]_{T_{\mathrm{dof}} \times N_{r}}, }
\end{aligned}
$$

in which $\mathbf{K}_{v_{j}-r_{k}}$ and $\mathbf{K}_{r_{k}-v_{j}}$ represent the stiffness matrices induced by the interaction between the $k$ th wheelset of the $j$ th trailer car and rail, and $\mathbf{C}_{v_{j}-r_{k}}$ and $\mathbf{C}_{r_{k}-v_{j}}$ are the corresponding damping matrices. $\mathbf{K}_{v_{j}-r_{1}}, \mathbf{K}_{v_{j}-r_{2}}, \mathbf{C}_{v_{j}-r_{1}}$, and $\mathbf{C}_{v_{j}-r_{2}}$ consist of zero row vectors except for those corresponding to the two DOFs of front bogie of the $j$ th trailer car, while $\mathbf{K}_{r_{1}-v_{j}}$, $\mathbf{K}_{r_{2}-v_{j}}, \mathbf{C}_{r_{1}-v_{j}}$, and $\mathbf{C}_{r_{2}-v_{j}}$ consist of similar column vectors. Accordingly, $\mathbf{K}_{v_{j}-r_{3}}, \mathbf{K}_{v_{j}-r_{4}}, \mathbf{C}_{v_{j}-r_{3}}, \mathbf{C}_{v_{j}-r_{4}}, \mathbf{K}_{r_{3}-v_{j}}, \mathbf{K}_{r_{4}-v_{j}}, \mathbf{C}_{r_{3}-v_{j}}$, and $\mathbf{C}_{r_{4}-v_{j}}$ are formed by row or column vectors where the only nonzero elements correspond to the two DOFs of the rear bogie.

The matrices induced by the interaction between motor car and rail can be worked out similarly.

The matrices for rail-slab-bridge interaction can be also derived by following the similar procedure for derivation of train-rail interaction matrices. The matrices for rail-slab interaction, induced by the matrices of fastener between rail and slab, are marked with subscript " $r s$ " or " $s r$." The matrices for slab-bridge interaction, induced by the matrices of CA layer between slab and bridge girder, are marked with subscript "sb" or "bs."

3.5. Load Vector for Train, Rail, Slab, and Bridge. The load vector $\mathbf{F}_{t}$ of train with order $V_{\text {dof }} \times 1$ can be written as

$$
\begin{gathered}
\mathbf{F}_{t}=\left[\begin{array}{lll}
\mathbf{F}_{t}^{1} & \mathbf{F}_{t}^{2}
\end{array}\right]^{\mathbf{T}}, \\
\mathbf{F}_{t}^{1}=\left[\begin{array}{llllll}
\mathbf{F}_{J 1}^{1} & \mathbf{F}_{v 1}^{1} & \mathbf{F}_{v 2}^{1} & \cdots & \mathbf{F}_{v N_{v}}^{1} & \mathbf{F}_{J 2}^{1}
\end{array}\right], \\
\mathbf{F}_{t}^{2}=\left[\begin{array}{llllll}
\mathbf{F}_{J 1}^{2} & \mathbf{F}_{v 1}^{2} & \mathbf{F}_{v 2}^{2} & \cdots & \mathbf{F}_{v N_{v}}^{2} & \mathbf{F}_{J 2}^{2}
\end{array}\right],
\end{gathered}
$$


where the load vector of the $j$ th trailer car $\mathbf{F}_{v j}^{1}$ and $\mathbf{F}_{v j}^{2}$ and the load vector of the $i$ th motor car $\mathbf{F}_{J i}^{1}$ and $\mathbf{F}_{J i}^{2}$ with order $6 \times 1$ can be written, respectively, as

$$
\begin{aligned}
& \mathbf{F}_{v j}^{1}=\left\{\begin{array}{c}
0 \\
0 \\
k_{p} r\left(x_{j 1}\right)+k_{p} r\left(x_{j 2}\right) \\
k_{p} L_{t} r\left(x_{j 1}\right)-k_{p} L_{t} r\left(x_{j 2}\right) \\
k_{p} r\left(x_{j 3}\right)+k_{p} r\left(x_{j 4}\right) \\
k_{p} L_{t} r\left(x_{j 3}\right)-k_{p} L_{t} r\left(x_{j 4}\right)
\end{array}\right\}^{\mathrm{T}}=\sum_{h=1}^{4} k_{p} r\left(x_{j h}\right) R_{v h}, \\
& \mathbf{F}_{v j}^{2}=\left\{\begin{array}{c}
0 \\
0 \\
c_{p} \dot{r}\left(x_{j 1}\right)+c_{p} \dot{r}\left(x_{j 2}\right) \\
c_{p} L_{t} \dot{r}\left(x_{j 1}\right)-c_{p} L_{t} \dot{r}\left(x_{j 2}\right) \\
c_{p} \dot{r}\left(x_{j 3}\right)+c_{p} \dot{r}\left(x_{j 4}\right) \\
c_{p} L_{t} \dot{r}\left(x_{j 3}\right)-c_{p} L_{t} \dot{r}\left(x_{j 4}\right)
\end{array}\right\}^{\mathrm{T}}=\sum_{h=1}^{4} c_{p} \dot{r}\left(x_{j h}\right) R_{v h}, \\
& \mathbf{F}_{J i}^{1}=\left\{\begin{array}{c}
0 \\
0 \\
k_{J p} r\left(x_{i 1}\right)+k_{I p} r\left(x_{i 2}\right) \\
k_{J p} L_{J t}^{r} r\left(x_{i 1}\right)-k_{J p} L_{J t} r\left(x_{i 2}\right) \\
k_{J p} r\left(x_{i 3}\right)+k_{I p} r\left(x_{i 4}\right) \\
k_{J p} L_{J t} r\left(x_{i 3}\right)-k_{J p} L_{J t} r\left(x_{i 4}\right)
\end{array}\right\}^{\mathrm{T}}=\sum_{h=1}^{4} k_{I P} r\left(x_{i h}\right) R_{J h}, \\
& \mathbf{F}_{J i}^{2}=\left\{\begin{array}{c}
0 \\
0 \\
c_{J p} \dot{r}\left(x_{i 1}\right)+c_{I p} \dot{r}\left(x_{i 2}\right) \\
c_{I p} L_{J t} \dot{r}\left(x_{i 1}\right)-c_{I p} L_{J J} \dot{r}\left(x_{i 2}\right) \\
c_{I p} \dot{r}\left(x_{i 3}\right)+c_{I p} \dot{r}\left(x_{i 4}\right) \\
c_{I p} L_{J t} \dot{r}\left(x_{i 3}\right)-c_{I p} L_{J t} \dot{r}\left(x_{i 4}\right)
\end{array}\right\}^{\mathrm{T}}=\sum_{h=1}^{4} c_{I p} \dot{r}\left(x_{i h}\right) R_{J h}, \\
& R_{v 1}=\left[\begin{array}{llllll}
0 & 0 & 1 & L_{t} & 0 & 0
\end{array}\right], \\
& R_{v 2}=\left[\begin{array}{llllll}
0 & 0 & 1 & -L_{t} & 0 & 0
\end{array}\right] \text {, } \\
& R_{v 3}=\left[\begin{array}{llllll}
0 & 0 & 0 & 0 & 1 & L_{t}
\end{array}\right] \text {, } \\
& R_{v 4}=\left[\begin{array}{llllll}
0 & 0 & 0 & 0 & 1 & -L_{t}
\end{array}\right] \text {, } \\
& R_{J 1}=\left[\begin{array}{llllll}
0 & 0 & 1 & L_{J t} & 0 & 0
\end{array}\right] \text {, } \\
& R_{J 2}=\left[\begin{array}{llllll}
0 & 0 & 1 & -L_{J t} & 0 & 0
\end{array}\right] \text {, } \\
& R_{J 3}=\left[\begin{array}{llllll}
0 & 0 & 0 & 0 & 1 & L_{J t}
\end{array}\right] \text {, } \\
& R_{J 4}=\left[\begin{array}{llllll}
0 & 0 & 0 & 0 & 1 & -L_{J t}
\end{array}\right]
\end{aligned}
$$

in which $\dot{r}(x)$ denotes the first derivative of rail irregularity $r(x)$. as

The load vector of rail $\mathbf{F}_{r}$ with order $N_{r} \times 1$ can be written

$$
\mathbf{F}_{r}=\mathbf{F}_{r}^{0}+\mathbf{F}_{r}^{1}+\mathbf{F}_{r}^{2}+\mathbf{F}_{r}^{3}
$$

with

$$
\begin{gathered}
\mathbf{F}_{r}^{0}=\sum_{j=1}^{N_{v}} \sum_{h=1}^{4} \mathbf{F}_{r j h}^{0}+\sum_{i=1}^{2} \sum_{h=1}^{4} \mathbf{F}_{J r i h}^{0}, \\
\mathbf{F}_{r}^{1}=\sum_{j=1}^{N_{v}} \sum_{h=1}^{4} \mathbf{F}_{r j h}^{1}+\sum_{i=1}^{2} \sum_{h=1}^{4} \mathbf{F}_{J r i h}^{1}, \\
\mathbf{F}_{r}^{2}=\sum_{j=1}^{N_{v}} \sum_{h=1}^{4} \mathbf{F}_{r j h}^{2}+\sum_{i=1}^{2} \sum_{h=1}^{4} \mathbf{F}_{J r i h}^{2}, \\
\mathbf{F}_{r}^{3}=\sum_{j=1}^{N_{v}} \sum_{h=1}^{4} \mathbf{F}_{r j h}^{3}+\sum_{i=1}^{2} \sum_{h=1}^{4} \mathbf{F}_{J r i h}^{3}, \\
\mathbf{F}_{r j h}^{0}=g\left(m_{w}+\frac{1}{2} m_{t}+\frac{1}{4} m_{c}\right) \mathbf{N}_{j h}^{\mathrm{T}}, \\
\mathbf{F}_{r j h}^{1}=-k_{p} r\left(x_{j h}\right) \mathbf{N}_{j h}^{\mathrm{T}}, \\
\mathbf{F}_{r j h}^{2}=-c_{p} \dot{r}\left(x_{j h}\right) \mathbf{N}_{j h}^{\mathrm{T}}, \\
\mathbf{F}_{r j h}^{3}=-m_{w} \ddot{r}\left(x_{j h}\right) \mathbf{N}_{j h}^{\mathrm{T}}, \\
\mathbf{F}_{J r i h}^{0}=g\left(m_{J w}+\frac{1}{2} m_{J t}+\frac{1}{4} m_{J c}\right) \mathbf{N}_{J i h}^{\mathrm{T}}, \\
\mathbf{F}_{J r i h}^{1}=-k_{J p} r\left(x_{i h}\right) \mathbf{N}_{J i h}^{\mathrm{T}}, \\
\mathbf{F}_{J r i h}^{2}=-c_{J p} \dot{r}\left(x_{i h}\right) \mathbf{N}_{J i h}^{\mathrm{T}}, \\
\mathbf{F}_{J r i h}^{3}=-m_{J p} \ddot{r}\left(x_{i h}\right) \mathbf{N}_{J i h}^{\mathrm{T}},
\end{gathered}
$$

where $\ddot{r}(x)$ denotes the second derivative of rail irregularity $r(x)$ and $\mathbf{F}_{r j h}^{0}, \mathbf{F}_{r j h}^{1}, \mathbf{F}_{r j h}^{2}$, and $\mathbf{F}_{r j h}^{3}$, respectively, denote the load vectors of the $h$ th wheelset of the $j$ th trailer car acting upon the rail caused by gravity force of trailer car, rail irregularity, velocity of rail irregularity, and acceleration of rail irregularity. $\mathbf{F}_{\text {Irih }}^{0}, \mathbf{F}_{\text {Irih }}^{1}, \mathbf{F}_{\text {Irih }}^{2}$, and $\mathbf{F}_{\text {Irih }}^{3}$ are the load vectors of the $h$ th wheelset of the $i$ th motor car acting upon the rail caused by gravity force of motor car, rail irregularity, velocity of rail irregularity, and acceleration of rail irregularity, respectively.

In addition, each element for load vector of slab $\mathbf{F}_{s}$ with order $\bar{N}_{s} \times 1$ and load vector of bridge $\mathbf{F}_{b}$ with order $\bar{N}_{b} \times 1$ is zero.

Now let

$$
\mathbf{F}_{G}(t)=\left[\begin{array}{c}
\mathbf{0}_{V_{\mathrm{dof}} \times 1} \\
\mathbf{F}_{r}^{0} \\
\mathbf{0}_{\bar{N}_{s} \times 1} \\
\mathbf{0}_{\bar{N}_{b} \times 1}
\end{array}\right], \quad \mathbf{F}_{R}^{1}(t)=\left[\begin{array}{c}
\mathbf{F}_{t}^{1} \\
\mathbf{F}_{r}^{1} \\
\mathbf{0}_{\bar{N}_{s} \times 1} \\
\mathbf{0}_{\bar{N}_{b} \times 1}
\end{array}\right],
$$

$$
\mathbf{F}_{R}^{2}(t)=\left[\begin{array}{c}
\mathbf{F}_{t}^{2} \\
\mathbf{F}_{r}^{2} \\
\mathbf{0}_{\bar{N}_{s} \times 1} \\
\mathbf{0}_{\bar{N}_{b} \times 1}
\end{array}\right], \quad \mathbf{F}_{R}^{3}(t)=\left[\begin{array}{c}
\mathbf{0}_{V_{\mathrm{dof}} \times 1} \\
\mathbf{F}_{r}^{3} \\
\mathbf{0}_{\bar{N}_{s} \times 1} \\
\mathbf{0}_{\bar{N}_{b} \times 1}
\end{array}\right] \text {. }
$$


Then, the load vector of the total train-slab track-bridge interaction system $\mathbf{F}(t)$ can be expressed by the deterministic load vector $\mathbf{F}_{G}(t)$ induced by the train gravity force and the random load vector $\mathbf{F}_{R}(t)$ induced by the rail irregularity can be expressed as

$$
\begin{aligned}
\mathbf{F}(t) & =\left[\begin{array}{l}
\mathbf{F}_{t} \\
\mathbf{F}_{r} \\
\mathbf{F}_{s} \\
\mathbf{F}_{b}
\end{array}\right]=\mathbf{F}_{G}(t)+\mathbf{F}_{R}(t) \\
& =\mathbf{F}_{G}(t)+\mathbf{F}_{R}^{1}(t)+\mathbf{F}_{R}^{2}(t)+\mathbf{F}_{R}^{3}(t) .
\end{aligned}
$$

\section{Random Vibration Analysis of Train-Slab Track-Bridge Interaction System by PEM}

4.1. The Pseudoexcitation for the System. Let $x$ be the distance from the left-hand starting point of the track model to the wheel/rail contact point at time $t$; there exists a transformation from the PSD function $\mathrm{S}_{r r}(\Omega)$ of $r(x)$ in space domain to the PSD function $\mathbf{S}_{r r}(\omega)$ of $r(t)$ in time domain, according to $x=v t$ :

$$
\mathbf{S}_{r r}(\omega)=\frac{\mathbf{S}_{r r}(\Omega)}{v}
$$

where $\omega, \Omega$, and $v$ denote, respectively, the time frequency $(\mathrm{rad} / \mathrm{s})$, the spatial frequency $(\mathrm{rad} / \mathrm{m})$, and train speed $(\mathrm{m} / \mathrm{s})$. Obviously, $\omega=\Omega v$.

According to (21)-(26), the pseudoexcitation $\widetilde{\mathbf{F}}_{R}(\omega, t)$ induced by the rail irregularity can be obtained by PEM:

$$
\widetilde{\mathbf{F}}_{R}(\omega, t)=\widetilde{\mathbf{F}}_{R}^{1}(\omega, t)+\widetilde{\mathbf{F}}_{R}^{2}(\omega, t)+\widetilde{\mathbf{F}}_{R}^{3}(\omega, t)
$$

with

$$
\begin{aligned}
& \widetilde{\mathbf{F}}_{R}^{1}(\omega, t)=\left[\begin{array}{c}
\widetilde{\mathbf{F}}_{t}^{1}(\omega, t) \\
\widetilde{\mathbf{F}}_{r}^{1}(\omega, t) \\
\mathbf{0}_{\bar{N}_{s} \times 1} \\
\mathbf{0}_{\bar{N}_{b} \times 1}
\end{array}\right], \quad \widetilde{\mathbf{F}}_{R}^{2}(\omega, t)=\left[\begin{array}{c}
\widetilde{\mathbf{F}}_{t}^{2}(\omega, t) \\
\widetilde{\mathbf{F}}_{r}^{2}(\omega, t) \\
\mathbf{0}_{\bar{N}_{s} \times 1} \\
\mathbf{0}_{\bar{N}_{b} \times 1}
\end{array}\right], \\
& \widetilde{\mathbf{F}}_{R}^{3}(\omega, t)=\left[\begin{array}{c}
\mathbf{0}_{V_{\mathrm{dof}} \times 1} \\
\widetilde{\mathbf{F}}_{r}^{3}(\omega, t) \\
\mathbf{0}_{\bar{N}_{s} \times 1} \\
\mathbf{0}_{\bar{N}_{b} \times 1}
\end{array}\right] \\
& \widetilde{\mathbf{F}}_{t}^{1}(\omega, t)=\left[\begin{array}{llllll}
\widetilde{\mathbf{F}}_{J 1}^{1} & \widetilde{\mathbf{F}}_{v 1}^{1} & \widetilde{\mathbf{F}}_{v 2}^{1} & \cdots & \widetilde{\mathbf{F}}_{v N_{v}}^{1} & \widetilde{\mathbf{F}}_{J 2}^{1}
\end{array}\right]^{\mathbf{T}}, \\
& \mathbf{F}_{t}^{2}(\omega, t)=\left[\begin{array}{llllll}
\mathbf{F}_{J 1}^{2} & \mathbf{F}_{v 1}^{2} & \mathbf{F}_{v 2}^{2} & \cdots & \mathbf{F}_{v N_{v}}^{2} & \mathbf{F}_{J 2}^{2}
\end{array}\right]^{\mathbf{T}}, \\
& \widetilde{\mathbf{F}}_{v j}^{1}(\omega, t)=\sum_{h=1}^{4} k_{p} R_{v h} \sqrt{\mathbf{S}_{r r}(\omega)} e^{i \omega\left(t-t_{j h}\right)}, \\
& \widetilde{\mathbf{F}}_{J i}^{1}(\omega, t)=\sum_{h=1}^{4} k_{J p} R_{J h} \sqrt{\mathbf{S}_{r r}(\omega)} e^{\mathbf{i} \omega\left(t-t_{J h}\right)},
\end{aligned}
$$

$$
\begin{aligned}
\widetilde{\mathbf{F}}_{v j}^{2}(\omega, t)=\mathbf{i} \omega \sum_{h=1}^{4} c_{p} R_{v h} \sqrt{\mathbf{S}_{r r}(\omega)} e^{\mathbf{i} \omega\left(t-t_{j h}\right)}, \\
\widetilde{\mathbf{F}}_{J i}^{2}(\omega, t)=\mathbf{i} \omega \sum_{h=1}^{4} c_{J p} R_{J h} \sqrt{\mathbf{S}_{r r}(\omega)} e^{\mathbf{i} \omega\left(t-t_{J h}\right)}, \\
\widetilde{\mathbf{F}}_{r}^{1}(\omega, t)=\sum_{j=1}^{N_{v}} \sum_{h=1}^{4} \widetilde{\mathbf{F}}_{r j h}^{1}+\sum_{i=1}^{2} \sum_{h=1}^{4} \widetilde{\mathbf{F}}_{J r i h}^{1}, \\
\widetilde{\mathbf{F}}_{r}^{2}(\omega, t)=\sum_{j=1}^{N_{v}} \sum_{h=1}^{4} \widetilde{\mathbf{F}}_{r j h}^{2}+\sum_{i=1}^{2} \sum_{h=1}^{4} \widetilde{\mathbf{F}}_{J r i h}^{2}, \\
\widetilde{\mathbf{F}}_{r}^{3}(\omega, t)=\sum_{j=1}^{N_{v}} \sum_{h=1}^{4} \widetilde{\mathbf{F}}_{r j h}^{3}+\sum_{i=1}^{2} \sum_{h=1}^{4} \widetilde{\mathbf{F}}_{J r i h}^{3}, \\
\widetilde{\mathbf{F}}_{r j h}^{1}(\omega, t)=-k_{p} \mathbf{N}_{j h}^{\mathbf{T}} \sqrt{\mathbf{S}_{r r}(\omega)} e^{\mathbf{i} \omega\left(t-t_{j h}\right)}, \\
\widetilde{\mathbf{F}}_{r j h}^{2}(\omega, t)=-\mathbf{i} \omega c_{p} \mathbf{N}_{j h}^{\mathbf{T}} \sqrt{\mathbf{S}_{r r}(\omega)} e^{\mathbf{i} \omega\left(t-t_{j h}\right)}, \\
\widetilde{\mathbf{F}}_{J r j h}^{3}(\omega, t)=-\mathbf{i} \omega c_{J p} \mathbf{N}_{J i h}^{\mathbf{T}} \sqrt{\mathbf{S}_{r r}(\omega) e^{\mathbf{i} \omega\left(t-t_{J i h}\right)},} \\
\widetilde{\mathbf{F}}_{J r j}^{2}(\omega, t)=\omega^{2} m_{w} \mathbf{N}_{j h}^{\mathbf{T}} \sqrt{\mathbf{S}_{r r}(\omega)} e^{\mathbf{i} \omega\left(t-t_{j h}\right)}, \\
\widetilde{\mathbf{S}}^{2} m_{J w} \mathbf{N}_{J i h}^{\mathbf{T}} \sqrt{\mathbf{S}_{r r}(\omega)} e^{\mathbf{i} \omega\left(t-t_{J i h}\right)} .
\end{aligned}
$$

4.2. The Calculation of Random Responses of the System. Based on (1) and (26), the equations of motion for a train-slab track-bridge interaction system can be expressed as

$$
\mathbf{M}(t) \ddot{\mathbf{U}}+\mathbf{C}(t) \dot{\mathbf{U}}+\mathbf{C}(t) \mathbf{U}=\mathbf{F}_{G}(t)+\mathbf{F}_{R}(t)
$$

in which $\mathbf{M}(t), \mathbf{C}(t)$, and $\mathbf{K}(t)$ denote mass, damping, and stiffness matrices of the total train-slab track-bridge interaction system, respectively; $\mathbf{U}, \dot{U}$, and $\mathbf{U}$ denote, respectively, displacement, velocity and acceleration vectors of the system.

By assuming the rail irregularity to be a zero mean valued Gaussian random process, the mean value (MV) $\overline{\mathbf{Y}}_{\text {out }}(t)$ of arbitrary response of the system is only caused by the train gravity force and can be calculated easily by solving (31) in time domain using a step-by-step integration method. Consider

$$
\mathbf{M}(t) \overline{\ddot{\mathbf{U}}}+\mathbf{C}(t) \overline{\dot{\mathbf{U}}}+\mathbf{C}(t) \overline{\mathbf{U}}=\mathbf{F}_{G}(t) .
$$

The arbitrary pseudoresponse $\widetilde{\mathbf{Y}}_{\text {out }}(\omega, t)$ of the system caused by $\widetilde{\mathbf{F}}_{R}(\omega, t)$ can be obtained by solving (32) in frequency domain and time domain. Consider

$$
\mathbf{M}(t) \widetilde{\ddot{\mathbf{U}}}+\mathbf{C}(t) \widetilde{\dot{\mathbf{U}}}+\mathbf{C}(t) \widetilde{\mathbf{U}}=\widetilde{\mathbf{F}}_{R}(\omega, t) .
$$

According to PEM and random vibration theory, the time-dependent power spectral density (PSD) and standard 
TABLE 1: Parameters for trailer car and motor car.

\begin{tabular}{|c|c|c|c|c|}
\hline \multirow{2}{*}{ Notation } & \multirow{2}{*}{ Item } & \multirow{2}{*}{ Unit } & \multicolumn{2}{|c|}{ Value } \\
\hline & & & Trailer car & Motor car \\
\hline$m_{c} / m_{J c}$ & Mass of carbody & $\mathrm{kg}$ & $4.4 \times 10^{4}$ & $4.8 \times 10^{4}$ \\
\hline$J_{c} / J_{J c}$ & Mass moment of inertia of carbody & $\mathrm{kg} \cdot \mathrm{m}^{2}$ & $2.7 \times 10^{6}$ & $2.7 \times 10^{6}$ \\
\hline$m_{t} / m_{J t}$ & Mass of bogie & $\mathrm{kg}$ & $2.4 \times 10^{3}$ & $3.2 \times 10^{3}$ \\
\hline$J_{t} / J_{J t}$ & Mass moment of inertia of bogie & $\mathrm{kg} \cdot \mathrm{m}^{2}$ & $2.2 \times 10^{3}$ & $7.2 \times 10^{3}$ \\
\hline$m_{w} / m_{J w}$ & Mass of wheelset & $\mathrm{kg}$ & $2.4 \times 10^{3}$ & $2.4 \times 10^{3}$ \\
\hline$k_{s} / k_{J s}$ & Spring stiffness of the second suspension system & $\mathrm{N} / \mathrm{m}$ & $6.0 \times 10^{5}$ & $8.0 \times 10^{5}$ \\
\hline$c_{s} / c_{J s}$ & Damping coefficient of the second suspension system & $\mathrm{N} \cdot \mathrm{s} / \mathrm{m}$ & $8.0 \times 10^{4}$ & $9.0 \times 10^{4}$ \\
\hline$k_{p} / k_{J p}$ & Spring stiffness of the primary suspension system & $\mathrm{N} / \mathrm{m}$ & $1.4 \times 10^{6}$ & $2.08 \times 10^{6}$ \\
\hline$c_{p} / c_{J p}$ & Damping coefficient of the primary suspension system & $\mathrm{N} \cdot \mathrm{s} / \mathrm{m}$ & $8.0 \times 10^{4}$ & $6.0 \times 10^{4}$ \\
\hline$L_{v} / L_{J v}$ & Longitudinal distance between the center of bogie and the nearest side of carbody & $\mathrm{m}$ & 3.45 & 3.45 \\
\hline$L_{c} / L_{J c}$ & Half of longitudinal distance between the centers of front and rear bogies & $\mathrm{m}$ & 8.6875 & 8.6875 \\
\hline$L_{t} / L_{J t}$ & Half of bogie axle base & $\mathrm{m}$ & 1.25 & 1.25 \\
\hline$\omega_{c 1} / \omega_{J c 1}$ & The fundamental frequency for vertical motion of carbody & $\mathrm{Hz}$ & 0.75 & 0.84 \\
\hline$\omega_{c 2} / \omega_{I c 2}$ & The fundamental frequency for pitching motion of carbody & $\mathrm{Hz}$ & 0.84 & 0.97 \\
\hline$\omega_{t 1} / \omega_{J t 1}$ & The fundamental frequency for vertical motion of bogie & $\mathrm{Hz}$ & 6.00 & 4.78 \\
\hline$\omega_{t 2} / \omega_{I t 2}$ & The fundamental frequency for pitching motion of bogie & $\mathrm{Hz}$ & 7.10 & 6.28 \\
\hline
\end{tabular}

deviation (SD) of arbitrary response $\widetilde{\mathbf{Y}}_{\text {out }}(\omega, t)$ of the system can be written as

$$
\begin{aligned}
\mathbf{S}_{\text {Yout }}(\omega, t) & =\widetilde{\mathbf{Y}}_{\text {out }}(\omega, t) \widetilde{\mathbf{Y}}_{\text {out }}^{*}(\omega, t), \\
\sigma_{\text {Yout }}^{2}(t) & =\sum_{j=1}^{m} \mathbf{S}_{\text {Yout }}\left(\omega_{j}, t\right) \Delta \omega_{j},
\end{aligned}
$$

where the superscript "*" denotes complex conjugate, $m$ denotes the total number of discrete frequencies of rail irregularity, and $\Delta \omega_{j}$ denotes the $j$ th frequency interval of rail irregularity.

4.3. The Solution Procedures. The flowchart for efficiently analyzing the vertical random vibration of train-slab trackbridge interaction system under the action of rail irregularity is shown in Figure 2 by following the above explanation of random vibration analysis by PEM.

\section{Case Study}

5.1. The Properties of Train-Slab Track-Bridge Interaction System. In order to reduce the boundary effect of the subgrade as far as possible, a nine-span simply supported beam high-speed railway bridge with the span length of $32 \mathrm{~m}$ is considered as shown in Figure 1. The central part of railway slab track is supported on bridge, while the left and right parts of the track are supported on subgrades adjacent to the bridge. The track is assumed to be continuous throughout, while the lengths of left and right parts of track considered are, respectively, $260 \mathrm{~m}$ and $210 \mathrm{~m}$, in order that the train moving from left to right can not only reach the steady-state response [15] before it runs upon the track on bridge but also move fully out of the right boundary of bridge. The lengths of rail, slab, and bridge girder element are all equal to fastener spacing of $0.65 \mathrm{~m}$. The train comprises front and rear motor cars with the same properties and six identical trailer cars moving with constant velocity $v$. The physical parameters of train, track, and bridge are listed, respectively, in Tables 1 and 2. The Germany high-speed track vertical profile irregularity PSD function $[13,33]$ is adopted; that is,

$$
\mathbf{S}_{r r}(\Omega)=\frac{A_{v} \Omega_{c}^{2}}{\left(\Omega^{2}+\Omega_{r}^{2}\right)\left(\Omega^{2}+\Omega_{c}^{2}\right)},
$$

where $\Omega_{c}=0.8246 \mathrm{rad} / \mathrm{m}, \Omega_{r}=0.0206 \mathrm{rad} / \mathrm{m}$, and $A_{v}=$ $4.032 \times 10^{-7} \mathrm{~m} \cdot \mathrm{rad}$.

To solve the equations of motion for the train-slab trackbridge interaction system, the Wilson- $\theta$ method is used with $\theta=1.4$ and moving length of $0.1 \mathrm{~m}$ of vehicles along track for each time step. In addition, the spatial frequency of the PSD ranges from $0.004 \times 2 \pi$ to $1 \times 2 \pi \mathrm{rad} / \mathrm{m}$; that is to say, the wavelength of rail irregularity ranges from 1 to $250 \mathrm{~m}$. It should be noted that a series of wavelengths of unequal intervals are selected by the following expression; that is,

$$
W_{\text {Len }}(j)=C_{\text {Len }} e^{(j-1)} \quad(j=1,2,3, \ldots, m),
$$

where $W_{\text {Len }}$ denotes the wavelength of rail irregularity $(m)$ and $C_{\text {Len }}$ denotes a constant related to the maximum frequency points $m$.

\subsection{Selection of the Suitable Number of Discrete Frequencies} of Rail Irregularity. From the aforementioned solution procedures for random analysis of train-slab track-bridge system, one can find that the larger the number of discrete frequencies $m$ is selected, the more calculation steps are. In order to save computer time as much as possible and, meanwhile, ensure a high accuracy of solution, a sequence of numerical experimentations is carried out to obtain the suitable number of discrete frequencies of rail irregularity with train running at speed of $69.44 \mathrm{~m} / \mathrm{s}(250 \mathrm{~km} / \mathrm{h})$. As $m$ increases from 50 to 250 


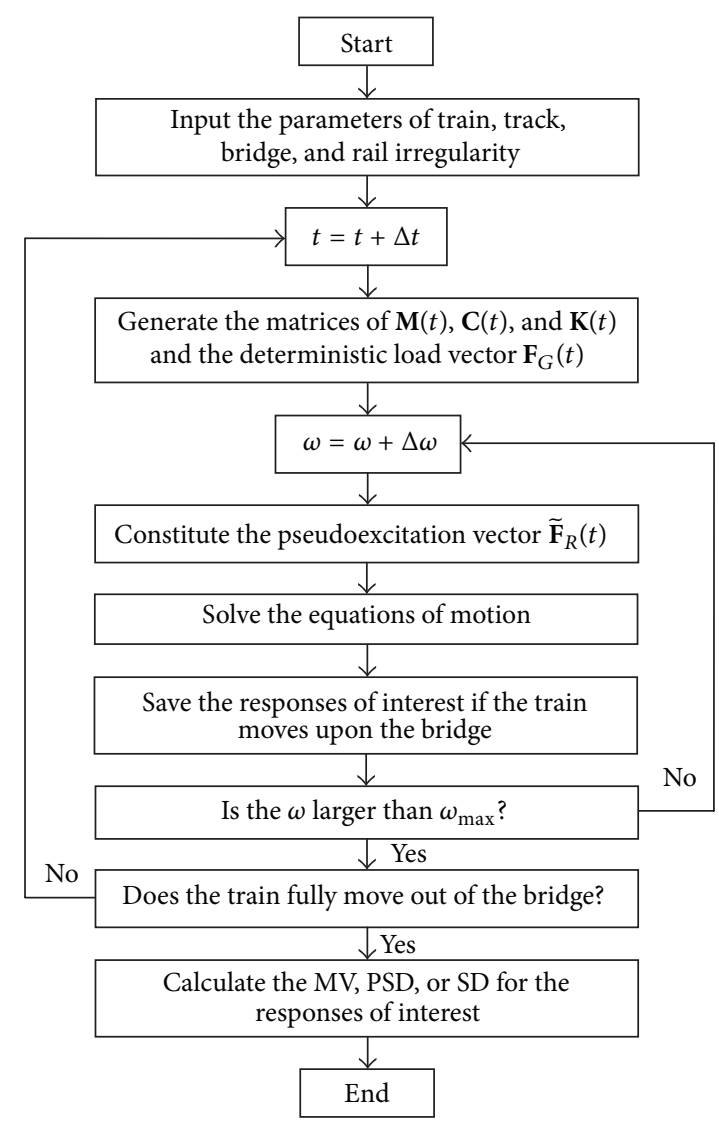

FIGURE 2: Flowchart for the vertical random vibration analysis of train-slab track-bridge interaction system.

TABLE 2: Parameters for slab track and bridge.

\begin{tabular}{clcc}
\hline Notation & Item & Unit & Value \\
\hline Slab track & & $\mathrm{N} / \mathrm{m}^{2}$ & $2.06 \times 10^{11}$ \\
$E_{r}$ & Young's modulus of rail & - & 0.3 \\
$v_{r}$ & Poisson's ratio of rail & $\mathrm{m}^{4}$ & $2 \times 3.217 \times 10^{-5}$ \\
$I_{r}$ & Mass moment of inertia of rail cross section & $\mathrm{kg} / \mathrm{m}$ & $2 \times 60.64$ \\
$\bar{m}_{r}$ & Mass per unit length of rail & $\mathrm{N} / \mathrm{m}^{2}$ & $3.6 \times 10^{10}$ \\
$E_{s}$ & Young's modulus of slab & - & 0.2 \\
$v_{s}$ & Poisson's ratio of slab & $\mathrm{m}^{4}$ & $1.6 \times 10^{-3}$ \\
$I_{s}$ & Mass moment of inertia of slab cross section & $\mathrm{kg} / \mathrm{m}$ & $1.2 \times 10^{3}$ \\
$\bar{m}_{s}$ & Mass per unit length of slab & $\mathrm{N} / \mathrm{m}$ & $2 \times 5.0 \times 10^{7}$ \\
$k_{r s}$ & Spring stiffness of fastener & $\mathrm{N} \cdot \mathrm{s} / \mathrm{m}$ & $2 \times 3.625 \times 10^{4}$ \\
$c_{r s}$ & Damping coefficient of fastener & $\mathrm{N} / \mathrm{m}^{2}$ & $2 \times 1.5 \times 10^{9}$ \\
$\bar{k}_{s b}$ & Spring stiffness of CA layer per unit length & & $2 \times 8.3 \times 10^{4}$ \\
$\bar{c}_{s b}$ & Damping coefficient of CA layer per unit length & $\mathrm{N} / \mathrm{m}^{2}$ & $3.45 \times 10^{10}$ \\
Bridge & & - & 0.2 \\
$E_{b}$ & Young's modulus of girder & $\mathrm{m}^{4}$ & 12.744 \\
$v_{b}$ & Poisson's ratio of girder & $\mathrm{kg} / \mathrm{m}$ & $2.972 \times 10^{4}$ \\
$I_{b}$ & Mass moment of inertia of girder cross section & - & 0.05 \\
$\bar{m}_{b}$ & Mass per unit length of bridge girder & $\mathrm{Hz}$ & 5.72 \\
$\zeta_{b}$ & Damping ratio of girder & $\mathrm{Hz}$ & 22.89 \\
$\omega_{b 1}$ & The fundamental frequency of girder & $\mathrm{N} / \mathrm{m}$ & $6.0 \times 10^{9}$ \\
$\omega_{b 2}$ & The second natural frequency of girder & $\mathrm{N} \cdot \mathrm{s} / \mathrm{m}$ & $2.0 \times 10^{6}$ \\
$k_{b b}$ & Spring stiffness of bearing & & \\
$c_{b b}$ & Damping coefficient of bearing & &
\end{tabular}


TABLE 3: Relationship between the number of discrete frequencies $m$ and the constant $C_{\text {Len }}$.

\begin{tabular}{|c|c|c|c|c|c|c|c|c|c|c|c|}
\hline$m$ & 50 & 70 & 90 & 110 & 130 & 150 & 170 & 190 & 210 & 230 & 250 \\
\hline$C_{\text {Len }}$ & 1.11929 & 1.08331 & 1.06342 & 1.05197 & 1.04374 & 1.03776 & 1.03322 & 1.02965 & 1.02677 & 1.02441 & 1.02243 \\
\hline
\end{tabular}

TABLE 4: The variation ratio $\mathrm{VR}_{1}$ of the responses (\%) and CPU times.

\begin{tabular}{|c|c|c|c|c|c|c|c|c|c|c|c|c|}
\hline \multirow[t]{2}{*}{ Item } & & \multicolumn{11}{|c|}{ Total number of discrete frequencies $m$} \\
\hline & & 50 & 70 & 90 & 110 & 130 & 150 & 170 & 190 & 210 & 230 & 250 \\
\hline \multirow{2}{*}{ Vertical acceleration of carbody } & Motor car & -1.99 & -1.29 & -0.88 & -0.62 & -0.47 & -0.34 & -0.23 & -0.16 & -0.08 & -0.03 & 0.00 \\
\hline & Trailer car & -1.97 & -1.30 & -0.86 & -0.63 & -0.44 & -0.35 & -0.25 & -0.16 & -0.10 & -0.03 & 0.00 \\
\hline \multirow{2}{*}{ Vertical acceleration of rear bogie } & Motor car & -2.02 & -1.27 & -0.90 & -0.56 & -0.45 & -0.35 & -0.28 & -0.17 & -0.10 & -0.05 & 0.00 \\
\hline & Trailer car & -2.33 & -1.45 & -0.98 & -0.62 & -0.56 & -0.35 & -0.28 & -0.17 & -0.10 & -0.04 & 0.00 \\
\hline \multirow{2}{*}{ Wheel/rail vertical force } & Motor car & -1.20 & -2.99 & -0.49 & -0.31 & -0.56 & -0.38 & -0.27 & -0.21 & -0.15 & -0.10 & 0.00 \\
\hline & Trailer car & -1.06 & -1.97 & -0.24 & -0.13 & -0.60 & -0.32 & -0.15 & -0.06 & -0.23 & -0.14 & 0.00 \\
\hline \multirow{2}{*}{ Vertical displacement of bridge midpoint } & Rail & 0.98 & 0.57 & 0.41 & 0.33 & 0.24 & 0.16 & 0.08 & 0.08 & 0.08 & 0.00 & 0.00 \\
\hline & Girder & -2.99 & -1.12 & -0.93 & -0.56 & -0.37 & -0.37 & -0.19 & -0.19 & 0.00 & 0.00 & 0.00 \\
\hline \multirow{3}{*}{ Vertical acceleration of bridge midpoint } & Rail & 9.40 & 6.21 & 4.33 & 3.08 & 2.26 & 1.63 & 1.15 & 0.77 & 0.47 & 0.22 & 0.00 \\
\hline & Slab & 12.26 & 9.67 & 6.60 & 4.28 & 3.30 & 2.27 & 1.59 & 1.05 & 0.62 & 0.32 & 0.00 \\
\hline & Girder & -0.38 & -1.76 & 11.42 & 3.14 & 1.38 & -0.69 & -0.19 & -0.69 & 0.00 & 0.06 & 0.00 \\
\hline Vertical fastener pressure & & 1.04 & 0.67 & 0.47 & 0.33 & 0.24 & 0.18 & 0.13 & 0.08 & 0.04 & 0.02 & 0.00 \\
\hline CPU times $(\mathrm{h})$ & & 0.60 & 0.80 & 0.96 & 1.13 & 1.31 & 1.43 & 1.64 & 1.84 & 2.03 & 2.23 & 2.41 \\
\hline
\end{tabular}

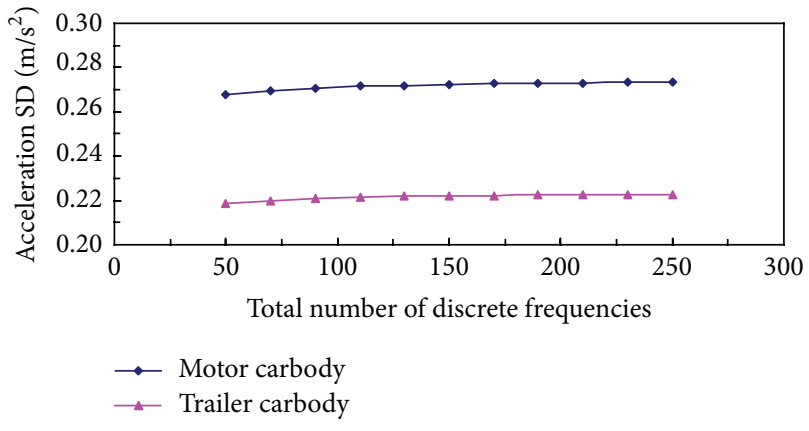

FIGURE 3: Maximum SD of vertical acceleration of carbody.

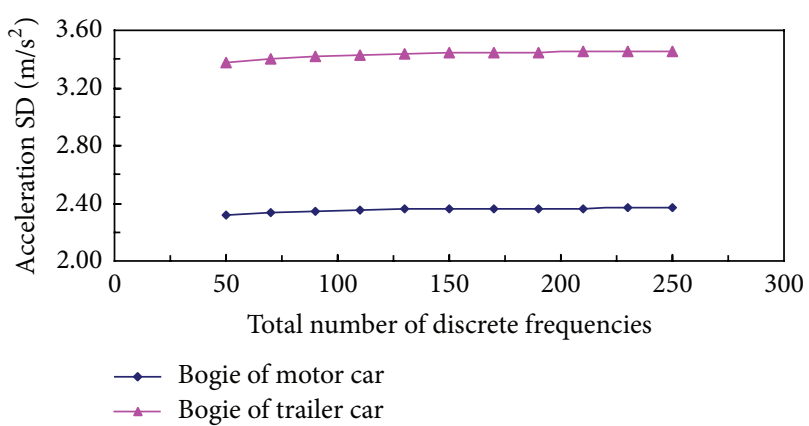

FIGURE 4: Maximum SD of vertical acceleration of rear bogie.

with increment 20 , the corresponding $C_{\text {Len }}$ is listed in Table 3 , based on which the solutions are plotted in Figures 3, 4, 5, 6, 7, and 8 . The variation ratio $\mathrm{VR}_{1}$ of the responses of train, track,

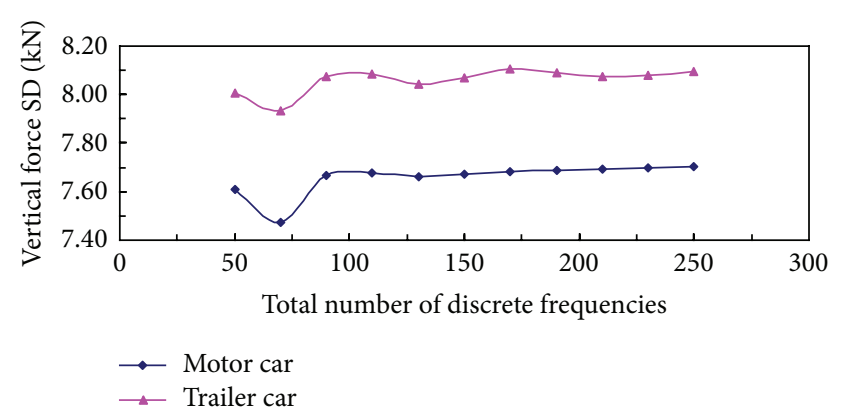

FIgure 5: Maximum SD of wheel/rail vertical force.

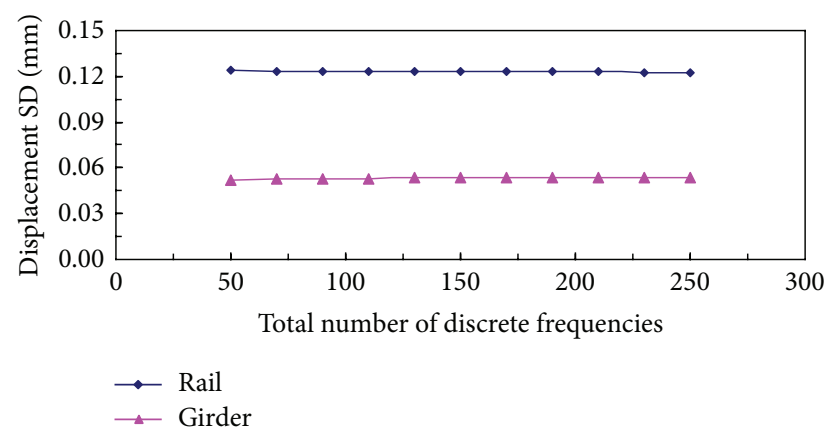

FIgURE 6: Maximum SD of vertical displacement of bridge midpoint.

and bridge and the CPU times for different cases are shown in Table 4. Herein $\mathrm{VR}_{1}$ is defined as (36), in which $R_{\mathrm{PEM}}(i)$ denote the maximum dynamic response SD of train, track, 


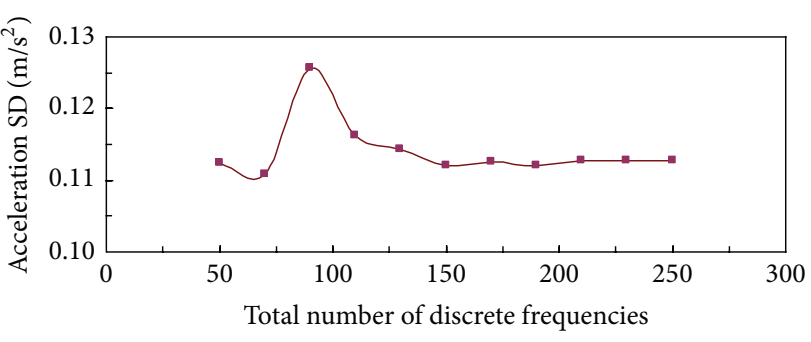

Figure 7: Maximum SD of vertical acceleration of bridge girder midpoint.

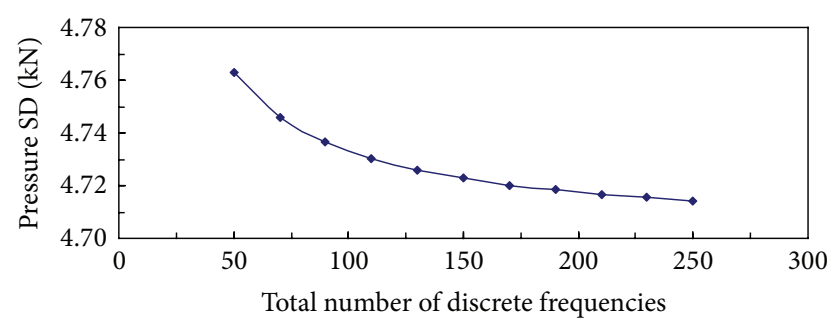

FIGURE 8: Maximum SD of vertical fastener pressure above bridge midpoint.

and bridge with the total number of discrete frequencies being equal to $i$ :

$$
\mathrm{VR}_{1}=\frac{R_{\mathrm{PEM}}(i)-R_{\mathrm{PEM}}(250)}{R_{\mathrm{PEM}}(250)} \times 100 \% \quad(i=50 \sim 250) .
$$

According to Yang et al. [15], the dynamic response of the last vehicle of a train should be given much more attention concerning the running safety and riding comfort of the train moving over a bridge because the last vehicle tends to vibrate more violently than the ones ahead. Therefore, "motor car" and "trailer car" considered in the following examples, respectively, mean the rear motor car and the $N_{v}$ th trailer car connecting with the rear motor car (Figure 1). In addition, "bridge midpoint" and "wheel/rail vertical force," respectively, are the midpoints of the fifth span for the ninespan bridge and the vertical contact force between the 3rd wheelset of the rear motor car or of the $N_{v}$ th trailer car and rail.

From Figures 3-8 and Table 4, it is observed that, as the total number of discrete frequencies increases, each of the maximum SD of train, track, and bridge dynamic responses tends to approach a limit value. Comparing the results obtained by 250 discrete frequencies with those by 170 discrete frequencies, the variation ratios $\mathrm{VR}_{1}$ are $-0.23 \%$ and $-0.25 \%$ for the vertical acceleration SD of the motor and trailer carbody, respectively, and $1.15 \%, 1.59 \%$, and $-0.19 \%$ for the vertical acceleration SD of rail, slab, and bridge girder, respectively. Therefore, one can conclude that the suitable number of discrete frequencies of rail irregularity can be equal to 170.
5.3. Comparing PEM with MCM. The running safety and riding comfort of train have been of great concern in railway engineering for a long time, particularly due to the development of high-speed railway. Several mechanisms that result in the derailment or discomfort of a running train have been identified through analytical and experimental investigations, based on which some indices have been proposed for evaluating the running safety and riding comfort of train [34], such as carbody vertical acceleration, wheel load decrement ratio, Sperling's ride index, and acceleration of bridge girder. In practice, the maximum dynamic responses are usually of the most interest for us. As is mentioned above, the conventional method of estimation to pick the maximum values from a set of response samples is extremely time consuming. However, the upper and lower boundaries of the responses by PEM can be estimated easily by the $\mathrm{MV} \pm 3$ times SD according to the $3 \sigma$ rule for Gaussian stochastic process [24], since the corresponding MV and SD can be obtained efficiently and accurately according to the aforementioned solution procedures.

For the purpose of comparison, the random responses of the train-slab track-bridge interaction system calculated by PEM, with total discrete frequencies $m=170$, are compared with those by MCM, with 1000 of rail irregularity samples. Herein, the method proposed by Chen and Zhai [35] is implemented to generate the rail irregularity sample $r(x)$ from the PSD function described as (34), and the train is also assumed to pass through the bridge with a constant speed $69.44 \mathrm{~m} / \mathrm{s}$. Figures $9,10,11,12,13,14,15,16,17,18,19$, and 20 show the estimated upper and lower boundaries of the responses by PEM and the corresponding extreme values of each time-history response obtained by MCM. The variation ratio $\mathrm{VR}_{2}$ of the responses of train, track, and bridge girder, determined according to (37), is shown in Table 5. Consider

$$
\begin{array}{r}
\mathrm{VR}_{2}=\frac{\max \left|R_{\mathrm{MCM}}^{E}(i)\right|-\min \left|R_{\mathrm{MCM}}^{E}(i)\right|}{\min \left|R_{\mathrm{MCM}}^{E}(i)\right|} \times 100 \% \\
(i=1 \sim 1000),
\end{array}
$$

where $R_{\mathrm{MCM}}^{E}$ denote the extreme values of each time-history response obtained by MCM and $i$ denotes the number of rail irregularity samples.

From Figures 9-20 and Table 5, the following observations can be made easily. (1) There exist dramatic variations for the extreme values of each time-history response by MCM, except for that of bridge girder vertical displacement. For example, variation ratio $\mathrm{VR}_{2}$ of vertical acceleration of motor carbody, wheel load decrement ratio of trailer car, Sperling's ride index of motor car, and vertical acceleration of bridge girder midpoint among the 1000 samples are $280.37 \%$, $120.08 \%, 39.00 \%$, and $170.05 \%$, respectively. In general, a larger number of rail irregularity samples tend to enlarge the variation ratio $\mathrm{VR}_{2}$. (2) All of extreme values of responses obtained by MCM fall with the corresponding limits of upper and lower boundaries of those by PEM. (3) Compared with MCM, the PEM helps to save computer time drastically. For example, the total CPU times for PEM with 170 discrete frequencies and MCM with just 100 samples are 1.64 hours 
TABLE 5: The variation ratio $\mathrm{VR}_{2}$ of the responses (\%) and CPU times.

\begin{tabular}{|c|c|c|c|c|c|c|c|}
\hline \multicolumn{2}{|l|}{ Item } & \multicolumn{6}{|c|}{ Total number of rail irregularity samples } \\
\hline & & 100 & 200 & 400 & 600 & 800 & 1000 \\
\hline \multirow{2}{*}{ Vertical acceleration of carbody } & Motor car & 152.25 & 193.00 & 228.30 & 228.30 & 280.37 & 280.37 \\
\hline & Trailer car & 150.17 & 150.17 & 158.91 & 160.20 & 184.66 & 184.66 \\
\hline \multirow{2}{*}{ Vertical acceleration of rear bogie } & Motor car & 61.94 & 74.82 & 79.93 & 98.72 & 98.72 & 98.72 \\
\hline & Trailer car & 62.92 & 69.90 & 83.86 & 112.07 & 112.07 & 112.07 \\
\hline \multirow{2}{*}{ Wheel load decrement ratio } & Motor car & 68.31 & 77.28 & 96.66 & 96.66 & 96.96 & 96.96 \\
\hline & Trailer car & 71.72 & 71.72 & 78.99 & 94.03 & 120.08 & 120.08 \\
\hline \multirow{2}{*}{ Sperling's ride index } & Motor car & 23.96 & 29.76 & 34.09 & 34.09 & 39.00 & 39.00 \\
\hline & Trailer car & 16.24 & 21.69 & 25.36 & 25.36 & 27.40 & 27.40 \\
\hline \multicolumn{2}{|c|}{ Vertical displacement of rail above bridge midpoint } & 49.74 & 60.51 & 61.94 & 61.94 & 68.28 & 68.28 \\
\hline \multicolumn{2}{|c|}{ Vertical fastener pressure above bridge midpoint } & 49.70 & 60.25 & 62.48 & 62.48 & 68.12 & 68.12 \\
\hline \multicolumn{2}{|c|}{ Vertical displacement of bridge girder midpoint } & 8.98 & 8.98 & 8.98 & 10.90 & 10.90 & 10.90 \\
\hline \multicolumn{2}{|c|}{ Vertical acceleration of bridge girder midpoint } & 111.92 & 115.58 & 160.73 & 160.73 & 160.73 & 170.05 \\
\hline \multicolumn{2}{|c|}{ CPU times $(\mathrm{h})$} & 13.12 & 26.24 & 52.48 & 78.72 & 91.84 & 131.20 \\
\hline
\end{tabular}

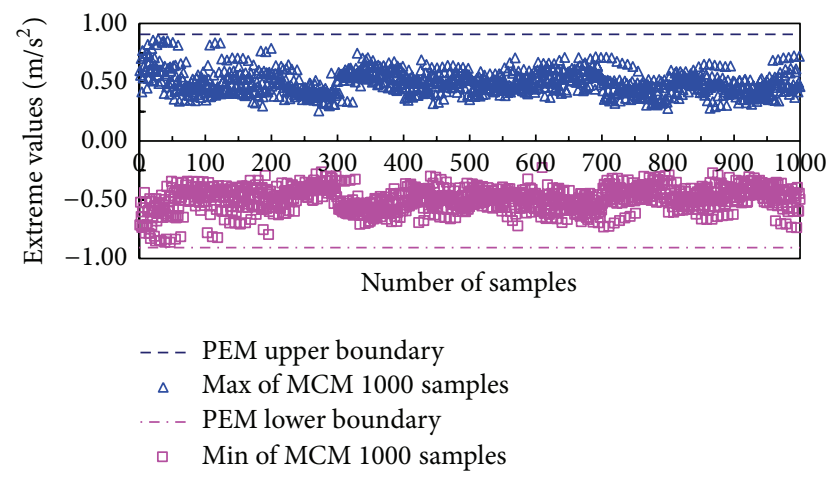

FIGURE 9: Comparison of vertical acceleration of motor carbody.

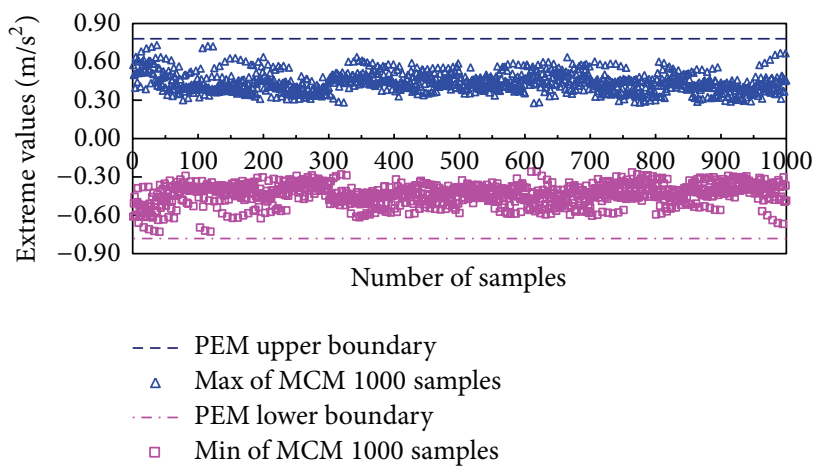

FIgURE 10: Comparison of vertical acceleration of trailer carbody.

and 13.12 hours, respectively, on a $2.8 \mathrm{GHz}$ personal computer. The latter equals about eight times the former. That is to say, PEM, in dealing with random vibration of train-slab trackbridge interaction system or evaluating the running safety and riding comfort of train, is not only more reliable but also more efficient than the conventional MCM.

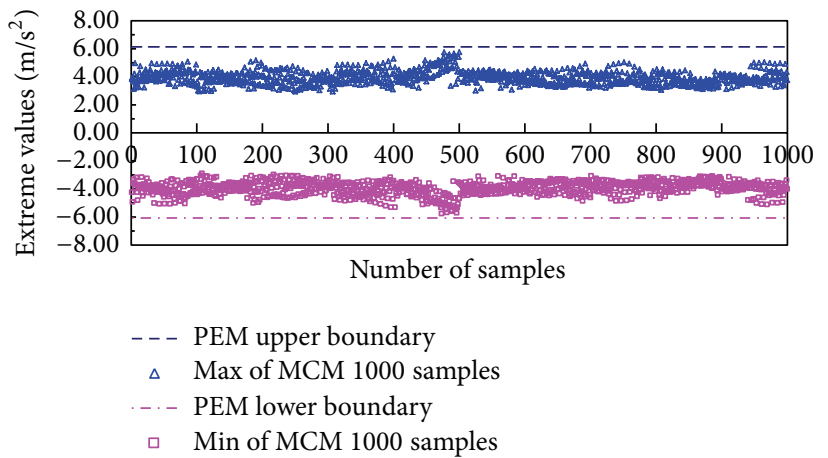

FIGURE 11: Comparison of rear bogie vertical acceleration of motor car.

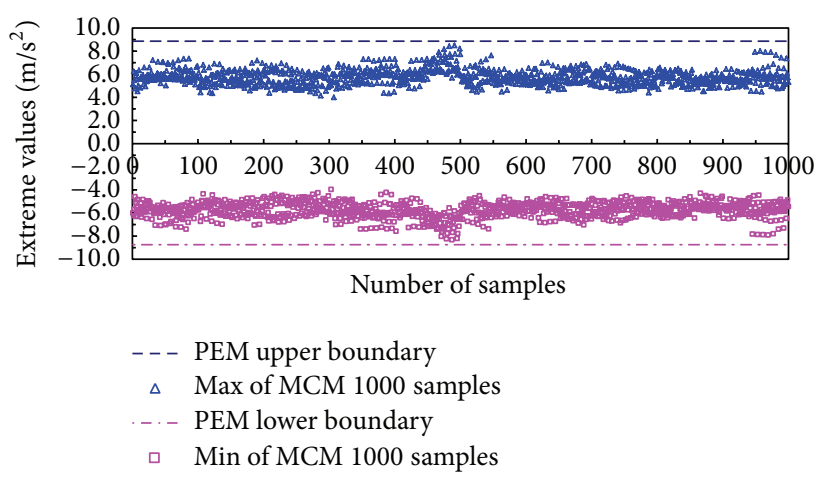

FIGURE 12: Comparison of rear bogie vertical acceleration of trailer car.

5.4. The Random Vibration Characteristics of Train-Slab Track-Bridge Interaction System. The dominant vibration frequency of train and bridge can be obtained easily according to the PSDs of dynamic responses calculated by PEM. To investigate the random vibration characteristics of train-slab 


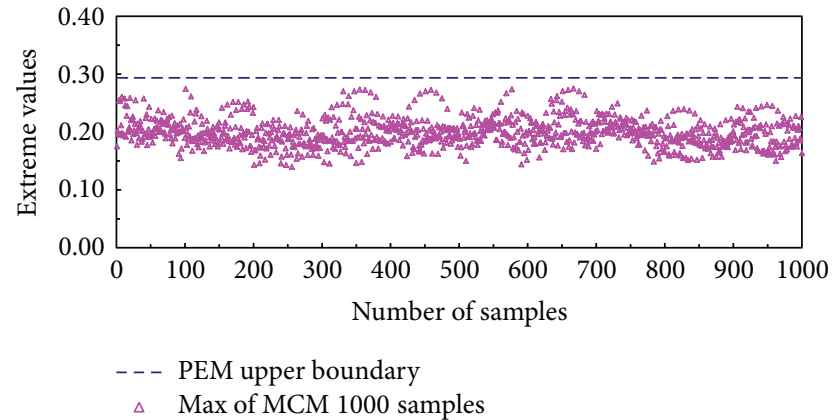

Figure 13: Comparison of wheel load decrement ratio of motor car.

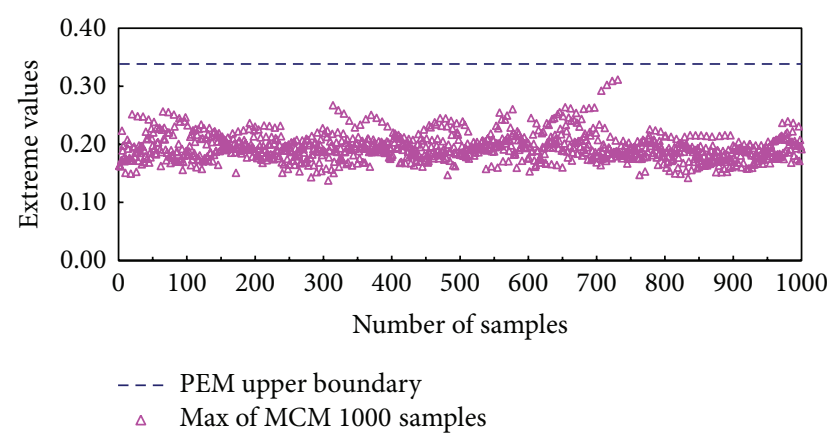

FIGURE 14: Comparison of wheel load decrement ratio of trailer car.

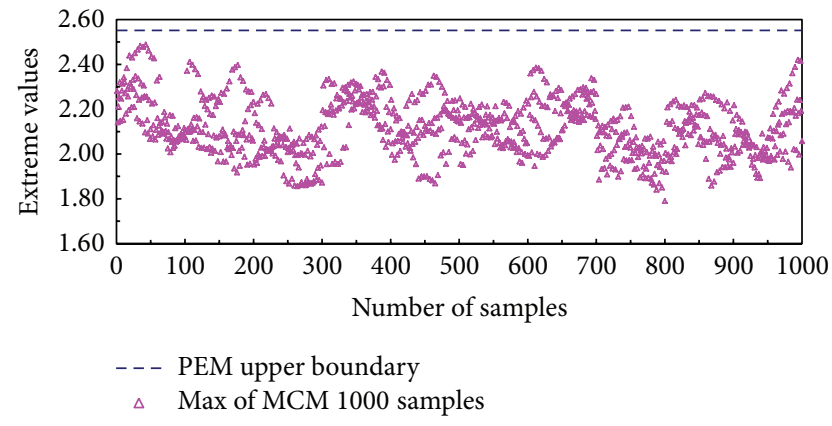

FIGURE 15: Comparison of Sperling's ride index of motor car.

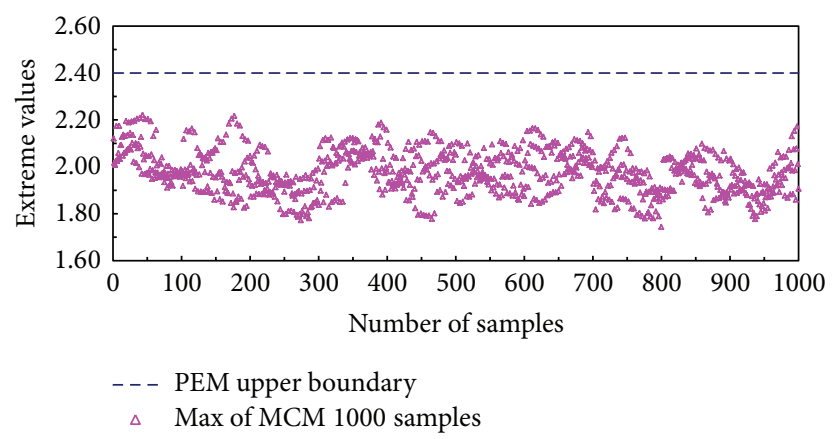

FIGURE 16: Comparison of Sperling's ride index of trailer car.

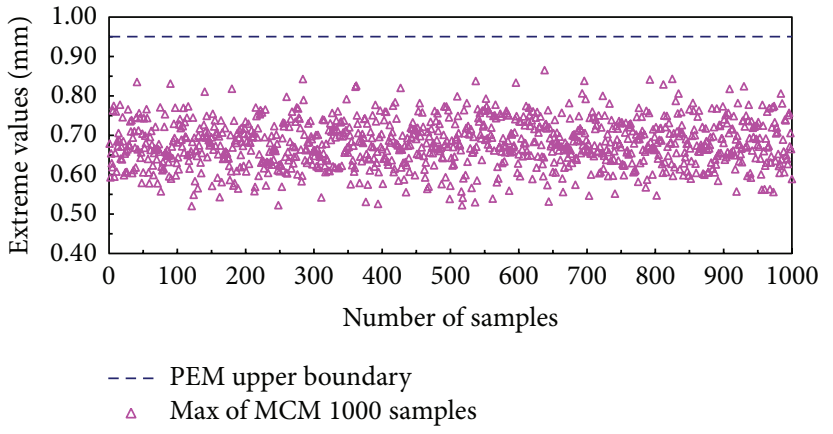

FIGURE 17: Comparison of vertical displacement of rail above bridge midpoint.

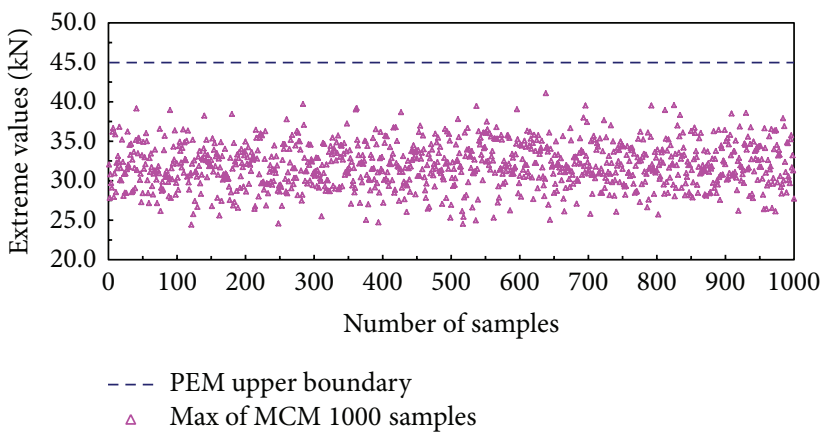

FIGURE 18: Comparison of vertical pressure of fastener above bridge midpoint.

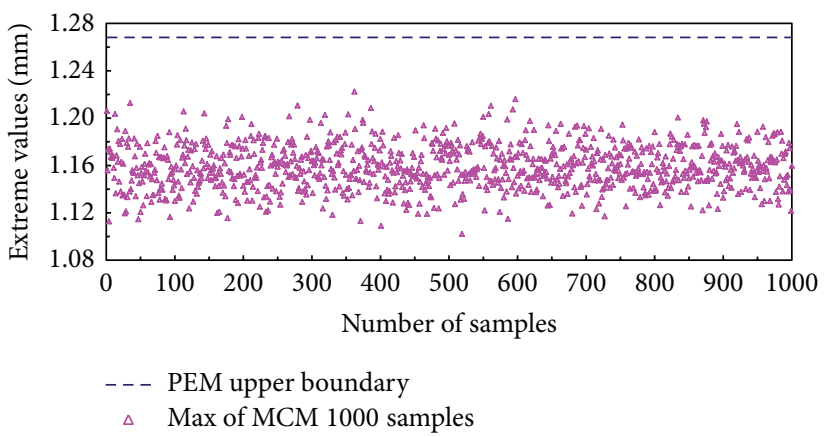

FIGURE 19: Comparison of vertical displacement of bridge girder midpoint.

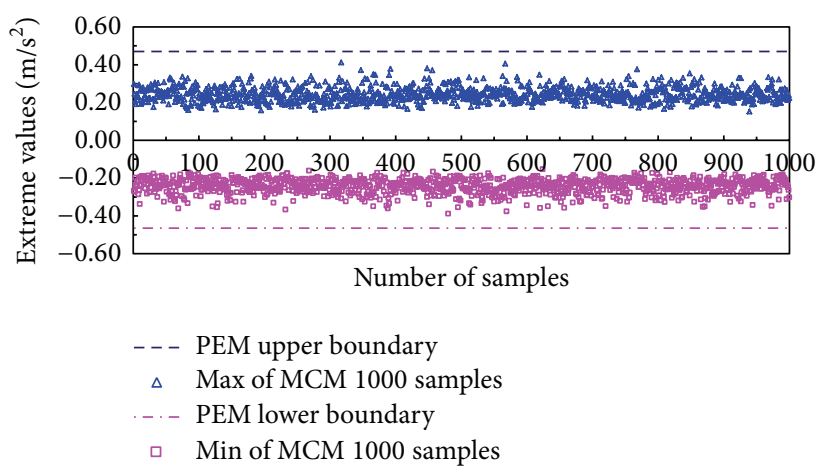

Figure 20: Comparison of vertical acceleration of bridge girder midpoint. 


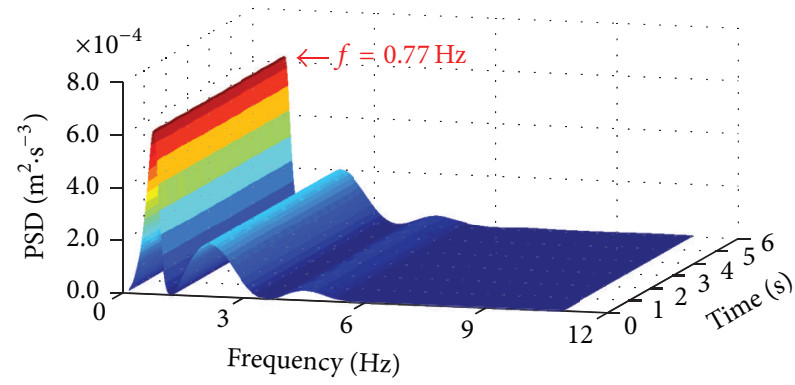

(a) $v=41.67 \mathrm{~m} / \mathrm{s}(150 \mathrm{~km} / \mathrm{h})$

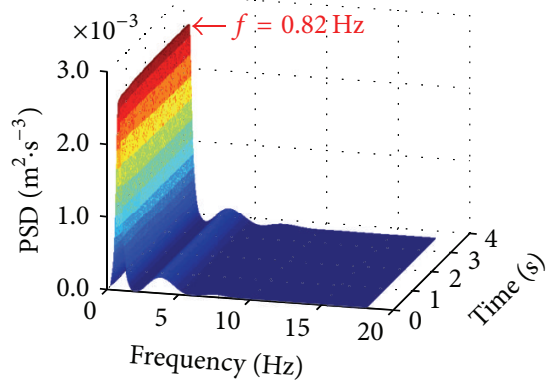

(b) $v=69.44 \mathrm{~m} / \mathrm{s}(250 \mathrm{~km} / \mathrm{h})$

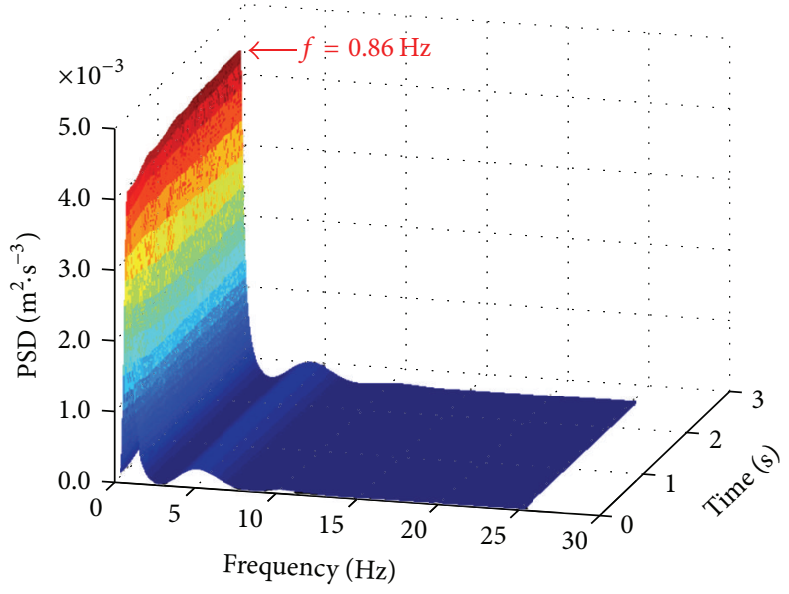

(c) $v=97.22 \mathrm{~m} / \mathrm{s}(350 \mathrm{~km} / \mathrm{h})$

FIGURE 21: PSD of vertical acceleration of motor carbody.

track-bridge interaction system, the solutions obtained by PEM, with 170 discrete frequencies of rail irregularity and three train speeds, are employed.

Figures 21 and 22 exhibit the PSDs of carbody vertical acceleration. As can be seen, the PSDs vary only slightly with time which indicates that the influence of bridge vibration on carbody vibration is insignificant because of the comparatively high mass and flexural rigidity of bridge girder. On the other hand, the PSDs change violently with rail irregularity frequency, implying the great influence of rail irregularity on the dynamic response of carbody. Of interest is that, no matter how the train speed changes, the peak values of PSDs of carbody vertical acceleration appear always approximately, respectively, to be around $0.77 \sim 0.86$ and $0.69 \sim 0.77 \mathrm{~Hz}$ for motor car and trailer car, which show good agreement with their own fundamental frequencies of 0.84 and $0.75 \mathrm{~Hz}$.

The similar phenomenon for bogie vertical acceleration can be also observed from Figures 23 and 24. As can be seen, the bogie also vibrates periodically, implying three dominant frequencies of $4.22,16.79$, and $33.49 \mathrm{~Hz}$ for $v=41.67 \mathrm{~m} / \mathrm{s}$, 6.31, 26.99, and $55.82 \mathrm{~Hz}$ for $v=69.44 \mathrm{~m} / \mathrm{s}$ and 6.37 (motor car), or 7.92 (trailer car), 39.18, and $78.15 \mathrm{~Hz}$ for $v=97.22 \mathrm{~m} / \mathrm{s}$. It is found that the vertical vibration frequency $f_{t}$ of bogie, the half of bogie axle base $L_{t}$, and the train speeds $v$ satisfy the relation $f_{t}=v /\left(n \times L_{t}\right), n=1,2,3, \ldots, \infty$, where relatively large contributions are made by harmonic components with $n=1,2$, or $8 \sim 15$.
The PSDs of vertical acceleration of bridge girder midpoint to the passage of the train are plotted in Figure 25. Similar with the cases of bogie vertical acceleration, there exist in Figure 25 three dominant frequency ranges of $4.7 \sim$ 5.4, 14 18, and $32 \sim 37 \mathrm{~Hz}$ for $v=41.67 \mathrm{~m} / \mathrm{s}, 4.4 \sim 6.3,25 \sim 33$, and $53 \sim 58 \mathrm{~Hz}$ for $v=69.44 \mathrm{~m} / \mathrm{s}$ and $4.4 \sim 6.8,35 \sim 43$, and $72 \sim 81 \mathrm{~Hz}$ for $v=97.22 \mathrm{~m} / \mathrm{s}$. Obviously, the first dominant frequency ranges of bridge girder vertical acceleration vary slightly around the fundamental frequency of bridge girder $\omega_{b 1}=5.72 \mathrm{~Hz}$ under various train speeds. However, the second and the third dominant frequency ranges increase as the train speed increases, both of whose trends quite coincide with those of bogie vertical acceleration (Figures 23 and 24). Furthermore, the contribution of the latter two ranges of dominant frequency to bridge girder vertical acceleration show an obvious trend of increase for higher train speeds.

From the point of view of structural dynamics, socalled resonance may occur if the wavelengths or frequencies implied by rail irregularity are close to the vehicle or bridge girder frequencies. Therefore, it is especially important to control strictly the rail irregularity with wavelengths of $v / \omega_{c 1}$ and $v / \omega_{b 1}$ in high-speed railway track maintenance, where $\omega_{c 1}$ and $\omega_{b 1}$ denote the fundamental frequencies of vertical motion of carbody and bridge girder, respectively. In addition, it is also significant to control the rail irregularity with wavelengths of $L_{t}$ and $2 L_{t}$ to reduce the vibration of 


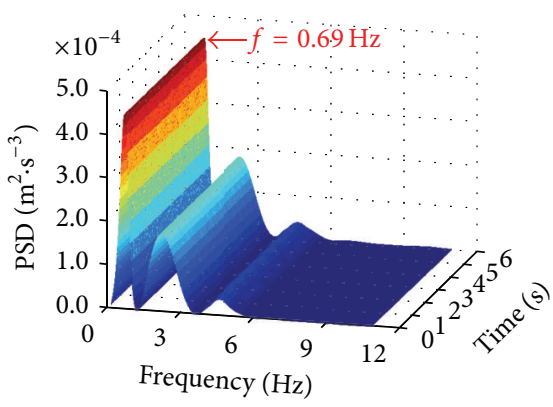

(a) $v=41.67 \mathrm{~m} / \mathrm{s}(150 \mathrm{~km} / \mathrm{h})$

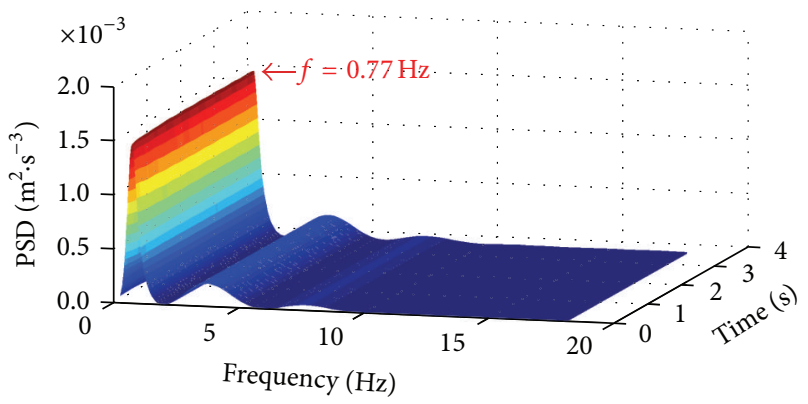

(b) $v=69.44 \mathrm{~m} / \mathrm{s}(250 \mathrm{~km} / \mathrm{h})$

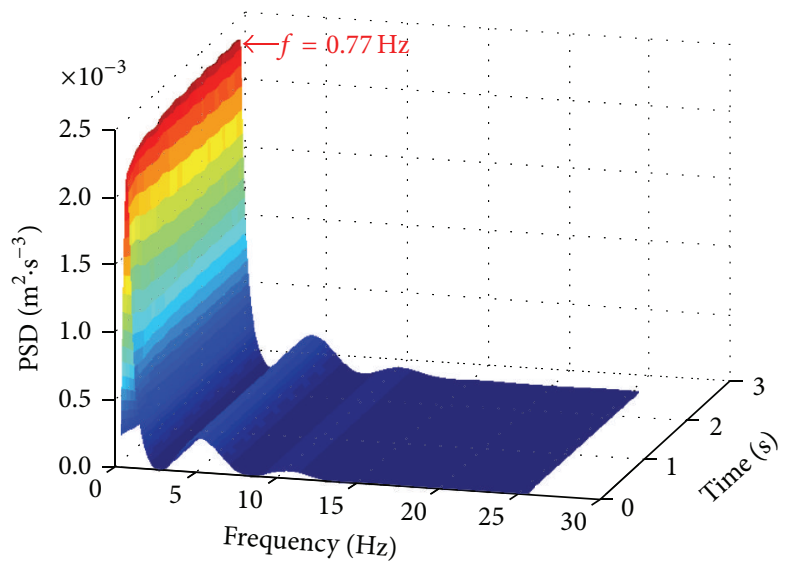

(c) $v=97.22 \mathrm{~m} / \mathrm{s}(350 \mathrm{~km} / \mathrm{h})$

FIGURE 22: PSD of vertical acceleration of trailer carbody.

bogie and bridge girder as much as possible for high-speed railway.

5.5. Influence of Train Speeds on the Dynamic Responses of Train-Slab Track-Bridge Interaction System. In reality, the train can move over the bridge at various speeds. There exists a need to investigate the dynamic responses of train-trackbridge system under various train speeds, as they may be quite different. The effect of train speeds on the dynamics responses of train-slab track-bridge interaction system is now investigated by PEM with speeds from 0 to $420 \mathrm{~km} / \mathrm{h}$ at $10 \mathrm{~km} / \mathrm{h}$ intervals and 170 discrete frequencies of rail irregularity considered. The estimated maximum responses of train, track, and bridge with respect to train speeds are shown in Figures 26, 27, 28, 29, and 30.

The maximum vertical accelerations of carbody under various train speeds are plotted in Figure 26. In general, the maximum response of the train moving over irregular track increases as train speeds increase, with a moderate growth for train speeds over $260 \mathrm{~km} / \mathrm{h}$. The reason for existence of such trend is that the exciting frequencies induced by rail irregularity at higher speeds are filtered out by the suspension system of each vehicle in transmission to the carbody. Moreover, both the maximum vertical accelerations of motor and trailer carbody are below the allowable limit of $1.3 \mathrm{~m} / \mathrm{s}^{2}$ imposed by China's provision [33] for speeds up to $420 \mathrm{~km} / \mathrm{h}$.
Figure 27 shows Sperling's ride index $W_{z}$ computed for the train under various speeds. Similar with the case of carbody vertical acceleration, Sperling's ride index of both motor car and trailer car increase comparatively slightly for train moving at moderate to high speeds $(200-420 \mathrm{~km} / \mathrm{h})$, with the former a mite higher than the latter. Compared with the allowable limit values set in China's provision for different comfort levels [33], Sperling's ride indices of motor car and trailer car fall with the limit of "tolerate $\left(2.75<W_{z}<3.0\right)$ " and "good $\left(2.5<W_{z}<2.75\right)$," respectively, under the track conditions specified for speeds up to $420 \mathrm{~km} / \mathrm{h}$.

Maximum wheel load decrement ratio with respect to train speeds are presented in Figure 28, which shows a trend of increase for higher train speeds. Of interest is the fact that the wheel load decrement ratio of trailer car is always greater than that of motor car. At the meantime, the former increases faster than the later for train speeds over $260 \mathrm{~km} / \mathrm{h}$. As a result, the wheel load decrement ratio of trailer car exceeds the limit of allowable 0.65 set in China's provision for the running safety of train [33] for speeds up to $370 \mathrm{~km} / \mathrm{h}$. In contrast, the wheel load decrement ratio of motor car remains to be lower than the allowable limit of 0.65 for speeds up to $420 \mathrm{~km} / \mathrm{h}$. From this point of view, the larger axle load is beneficial to ensure the running safety of train.

The impact responses of track and bridge girder traveled by train moving at speeds $0-420 \mathrm{~km} / \mathrm{h}$ are plotted in Figures 29 and 30, in which the impact factor $I$ is defined 


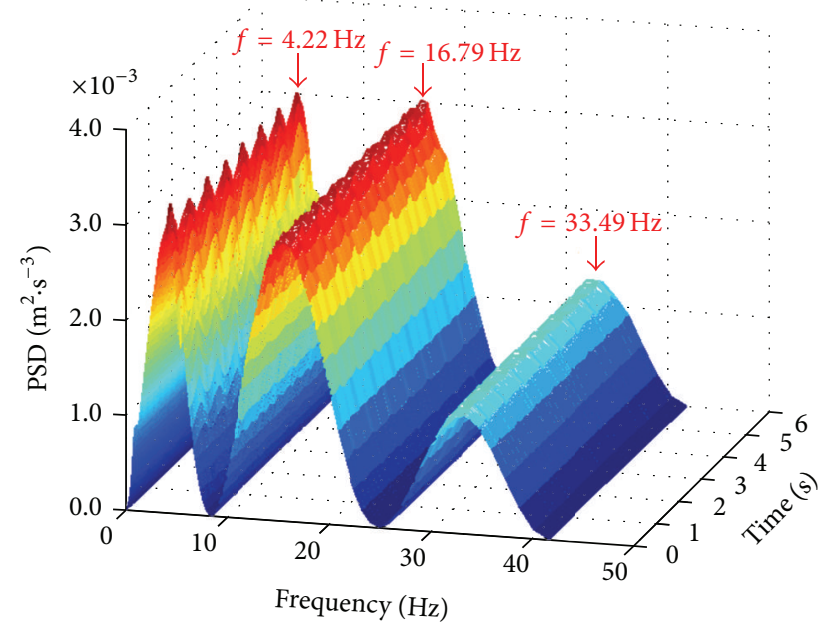

(a) $v=41.67 \mathrm{~m} / \mathrm{s}(150 \mathrm{~km} / \mathrm{h})$

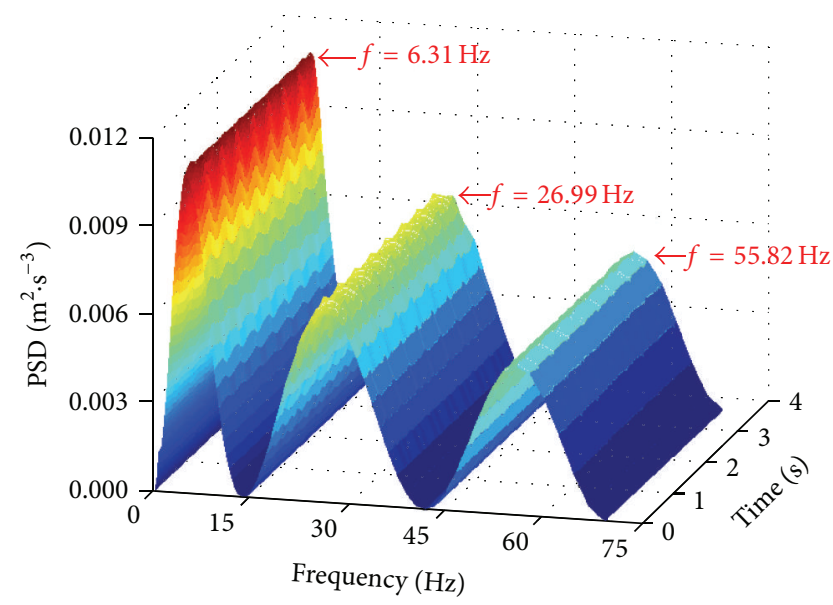

(b) $v=69.44 \mathrm{~m} / \mathrm{s}(250 \mathrm{~km} / \mathrm{h})$

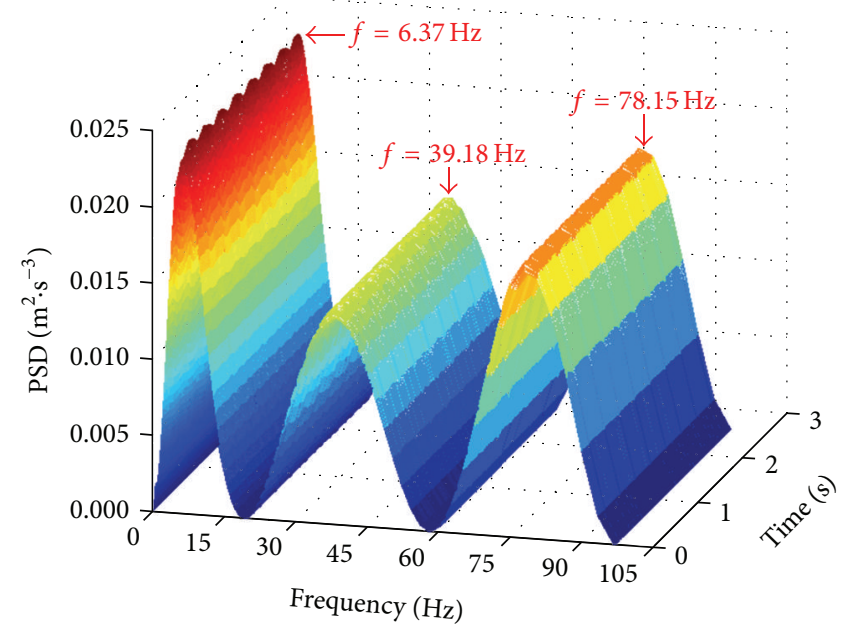

(c) $v=97.22 \mathrm{~m} / \mathrm{s}(350 \mathrm{~km} / \mathrm{h})$

FIGURE 23: PSD of vertical acceleration of motor car rear bogie.

as $I=\left[R_{d}(x)-R_{s}(x)\right] / R_{s}(x)$, where $R_{d}(x)$ and $R_{s}(x)$, respectively, denote the maximum dynamic and static vertical responses of track or bridge girder at section $x$ due to the action of train. As can be seen, the impact factor and vertical acceleration of bridge girder increase generally as the train speed increases, but no phenomenon of train-track-bridge resonance is observed, indicating that the fundamental frequency of bridge girder is suitable under the train properties specified for speeds over $420 \mathrm{~km} / \mathrm{h}$. In addition, the impact factor for rail vertical displacement is generally higher than that for bridge girder vertical displacement. The implication is that the extent of amplification for rail vertical displacement is larger than that for bridge girder vertical displacement, because of the comparatively small mass of rail and the effects of elasticity and damping of fastener and CA layer. Of interest is that the bridge girder vertical acceleration is far below the allowable limit of $3.5 \mathrm{~m} / \mathrm{s}^{2}$ set in China's provision under the track conditions specified for speed up to $420 \mathrm{~km} / \mathrm{h}$.

\section{Summary}

In this study, the vertical vibration model of train-slab trackbridge interaction system is firstly established by FEM; the equations of motion for the entire system are then derived by means of energy principle, and simultaneously the excitations between rail and wheels caused by random rail irregularity are transformed into a series of deterministic pseudoharmonic excitation vectors by PEM, and the random vibration responses of train, slab track, and bridge are finally obtained by step-by-step integration method. From the numerical results obtained in this work, the following conclusions can be drawn.

(1) In order to save computer time and ensure accuracy of solution as far as possible, some numerical experimentation should be done firstly to obtain the suitable number of discrete frequencies of rail irregularity in the process of random analysis of train-slab trackbridge interaction system. 


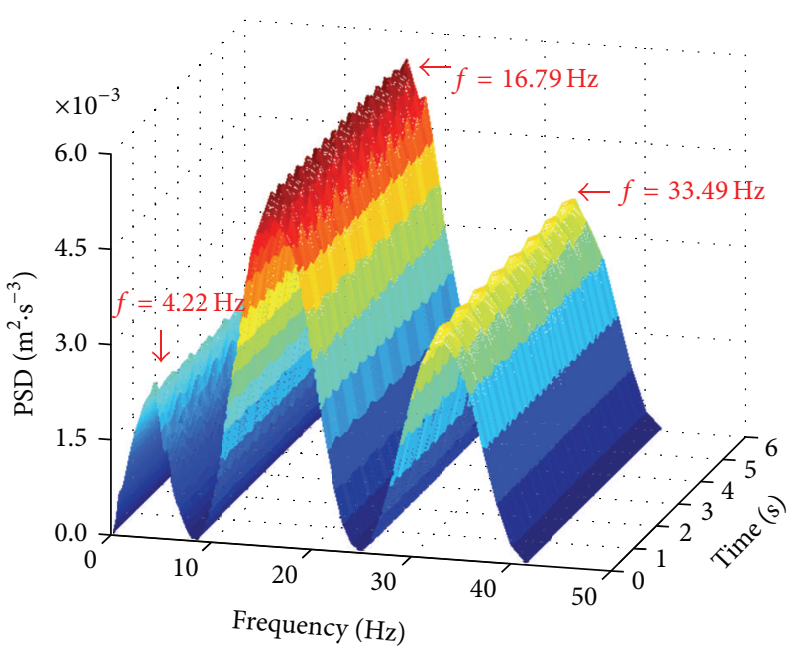

(a) $v=41.67 \mathrm{~m} / \mathrm{s}(150 \mathrm{~km} / \mathrm{h})$

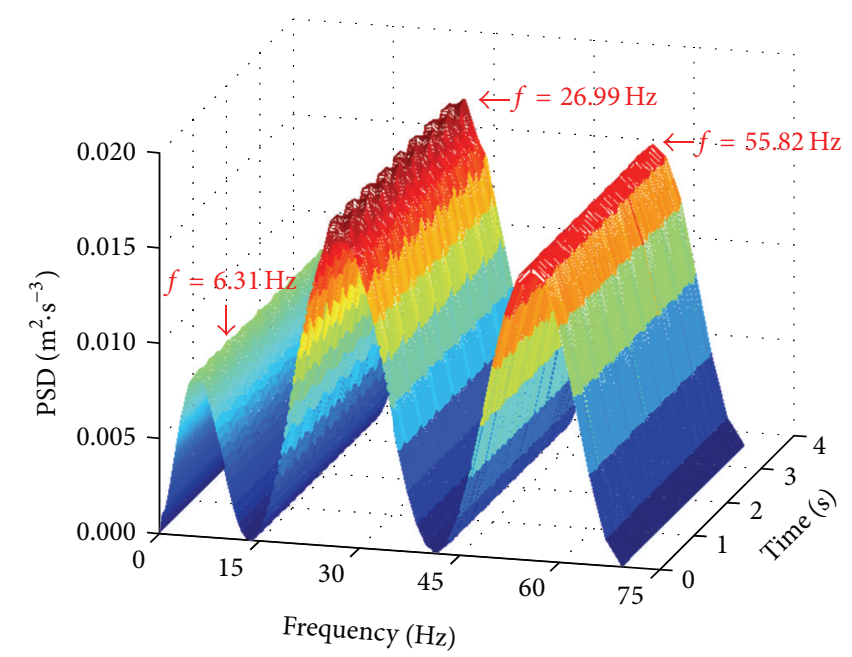

(b) $v=69.44 \mathrm{~m} / \mathrm{s}(250 \mathrm{~km} / \mathrm{h})$

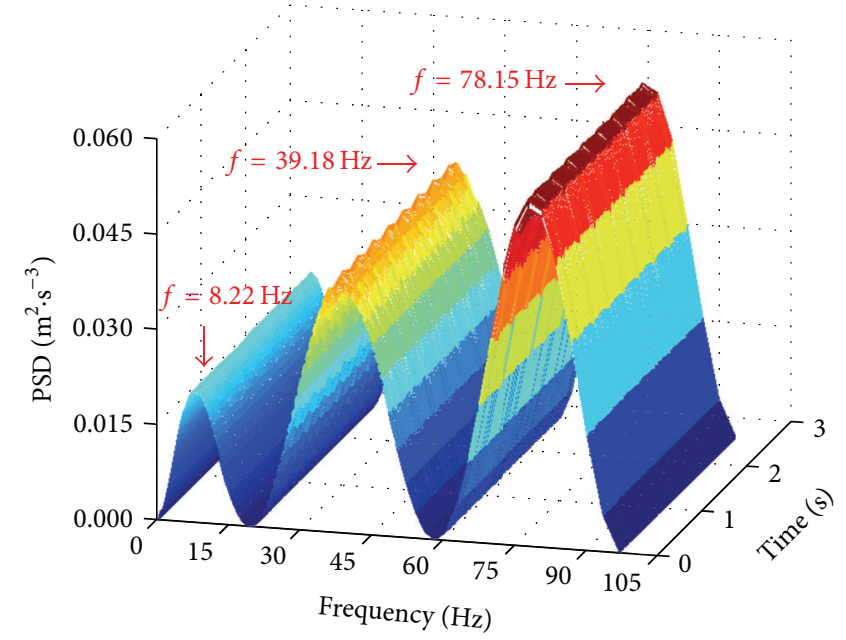

(c) $v=97.22 \mathrm{~m} / \mathrm{s}(350 \mathrm{~km} / \mathrm{h})$

FIGURE 24: PSD of vertical acceleration of trailer car rear bogie.

(2) The PEM can be applied not only to study the vertical random vibration characteristics of train-slab trackbridge interaction system but also to evaluate the running safety and riding comfort of train moving over bridge with various speeds, which is more reliable and efficient than the conventional MCM.

(3) The PEM may provide a new way to either optimize the design of dynamic parameters of train-slab trackbridge interaction system or provide guidance on the maintenance of track structure according to certain evaluation standard for railway.

(4) The equations of motion of vertical interaction system with other types of train, track, and bridge and the corresponding random vibration responses can be also obtained by the method proposed in this paper.

(5) In this work, only the vertical random vibration characteristics of train-slab track-bridge interaction system are investigated. In the future work, we plan on further developing the numerical method and extending its range of applicability to model the spatial random vibration characteristics of train-slab track-bridge interaction system.

\section{Conflict of Interests}

The authors declare that there is no conflict of interests regarding the publication of this paper.

\section{Acknowledgments}

The research summarized in this paper was supported by the Joint Funds of the National Natural Science Foundation of China through Grant nos. U1334203, U1361204, and U1134209, the National Natural Science Foundation of China through Grant no. 51378513, the Program for Changjiang 


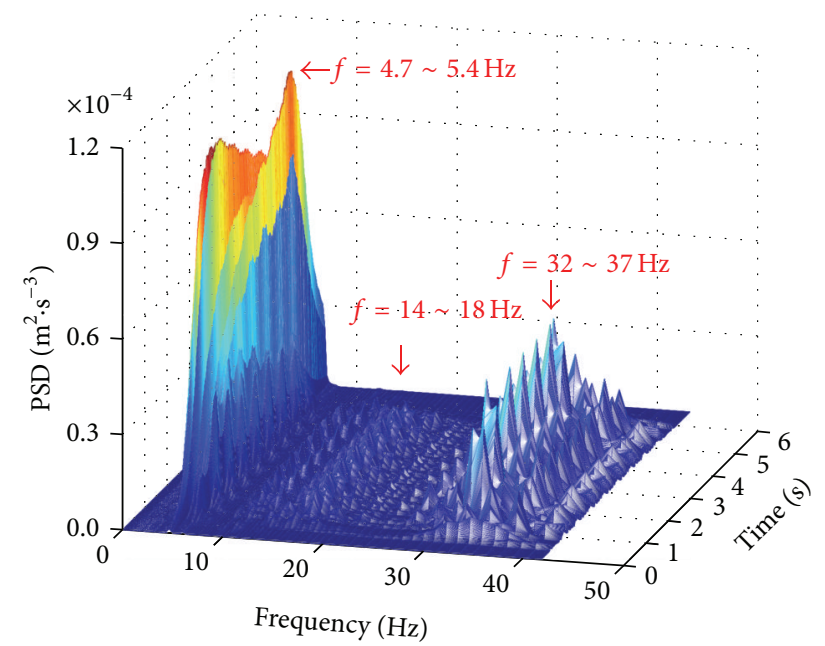

(a) $v=41.67 \mathrm{~m} / \mathrm{s}(150 \mathrm{~km} / \mathrm{h})$

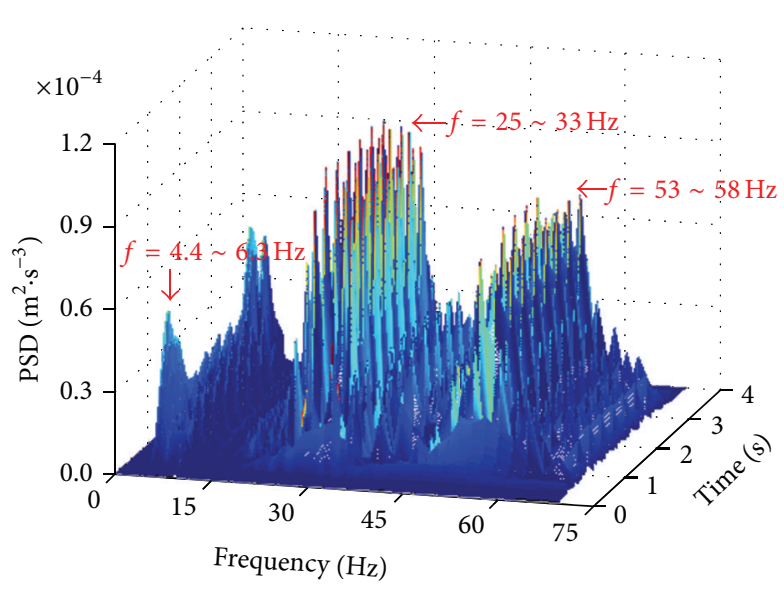

(b) $v=69.44 \mathrm{~m} / \mathrm{s}(250 \mathrm{~km} / \mathrm{h})$

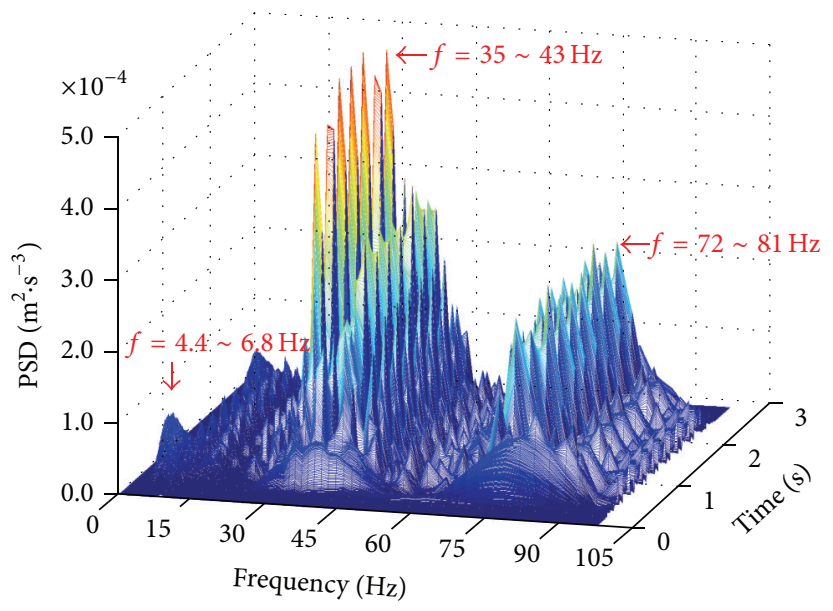

(c) $v=97.22 \mathrm{~m} / \mathrm{s}(350 \mathrm{~km} / \mathrm{h})$

FIGURE 25: PSD of vertical acceleration of bridge girder midpoint.

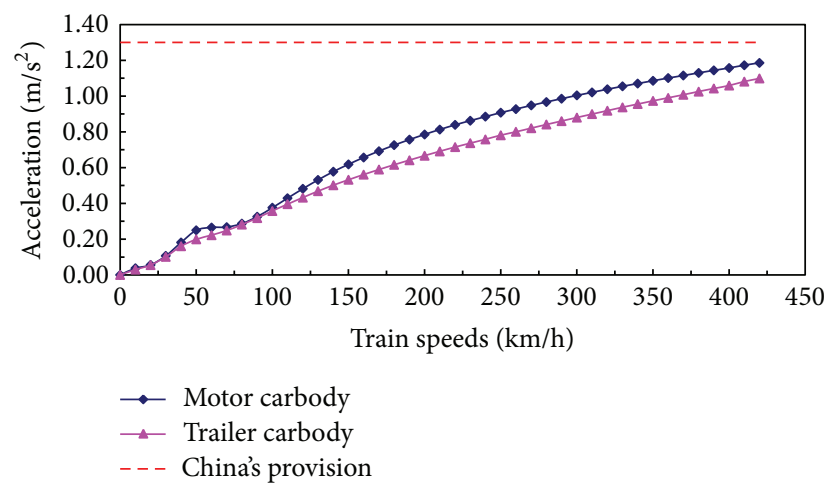

FIGURE 26: Maximum carbody vertical acceleration under various train speeds.

Scholars and Innovative Research Team in University through Grant no. IRT1296, the Science and Technology Foundation of Chinese Railway Corporation through Grants

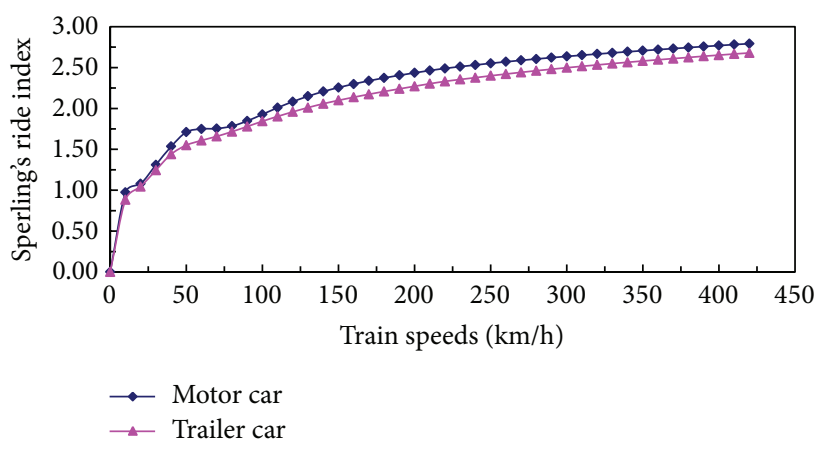

FIGURE 27: Maximum Sperling's ride index under various train speeds.

nos. 2012G013-B and 2013G008-E, the Teacher Research Fund of Central South University of China through Grant no. 2013JSJJ021, the China Postdoctoral Science Foundation through Grant no. 2013M530022, and the Major State Basic 


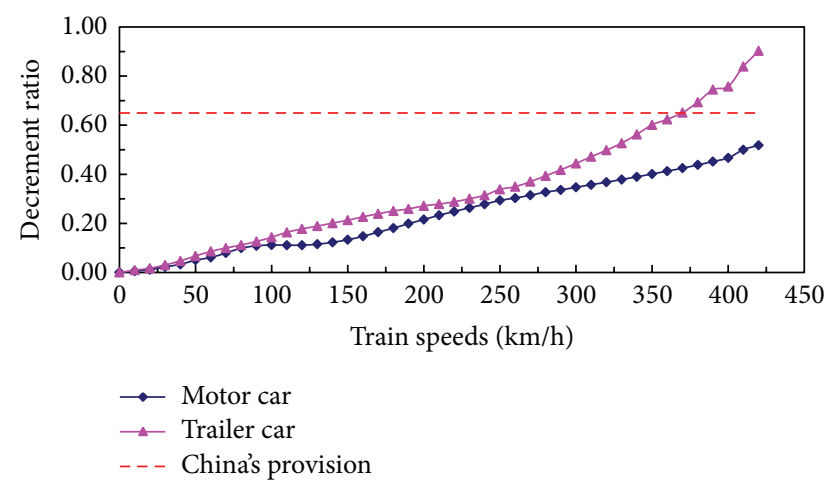

FIGURE 28: Maximum wheel load decrement ratio under various train speeds.

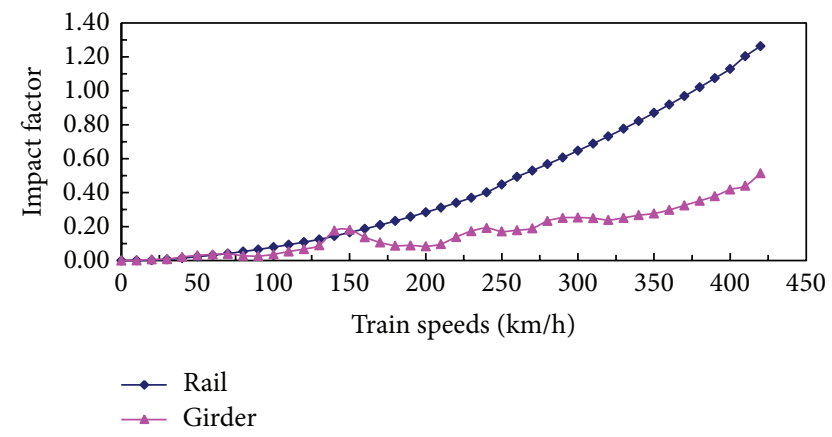

FIGURE 29: Maximum vertical impact factor of bridge midpoint under various train speeds (rail/girder).

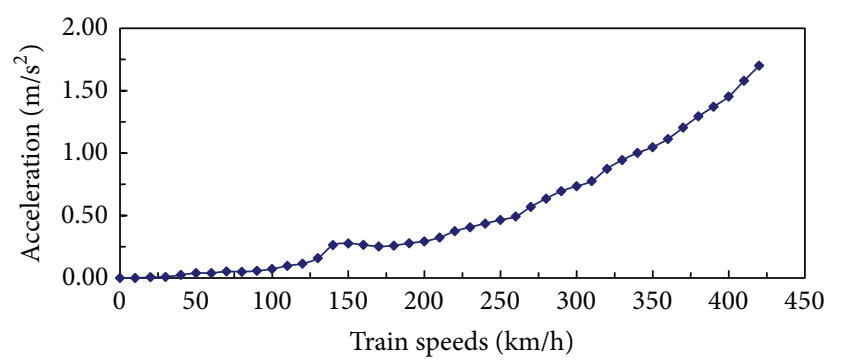

FIGURE 30: Maximum vertical acceleration of bridge girder midpoint under various train speeds.

Research Development Program of China through Grant no. 2013CB036203. These supports are gratefully acknowledged.

\section{References}

[1] K. H. Chu, V. K. Garg, and C. L. Dhar, "Railway-bridge impact: simplified train and bridge model," ASCE Journal of Structural Engineering, vol. 105, no. 9, pp. 1823-1844, 1979.

[2] R. A. Clark, P. A. Dean, J. A. Elkins, and S. G. Newton, "An investigation into the dynamic effects of railway vehicles running on corrugated rails," Proceedings of the Institution of Mechanical Engineers C: Journal of Mechanical Engineering Science, vol. 24, no. 2, pp. 65-76, 1982.
[3] G. Diana and F. Cheli, "Dynamic interaction of railway systems with large bridges," Vehicle System Dynamics, vol. 18, no. 1-3, pp. 71-106, 1989.

[4] O. Coussy, M. Said, and J. P. van Hoove, "The influence of random surface irregularities on the dynamic response of bridges under suspended moving loads," Journal of Sound and Vibration, vol. 130, no. 2, pp. 313-320, 1989.

[5] W. Zhai and Z. Cai, "Dynamic interaction between a lumped mass vehicle and a discretely supported continuous rail track," Computers \& Structures, vol. 63, no. 5, pp. 987-997, 1997.

[6] J. D. Yau, Y. B. Yang, and S. R. Kuo, "Impact response of high speed rail bridges and riding comfort of rail cars," Engineering Structures, vol. 21, no. 9, pp. 836-844, 1999.

[7] L. Frýba, "A rough assessment of railway bridges for high speed trains," Engineering Structures, vol. 23, no. 5, pp. 548-556, 2001.

[8] X. Lei and N.-A. Noda, "Analyses of dynamic response of vehicle and track coupling system with random irregularity of track vertical profile," Journal of Sound and Vibration, vol. 258, no. 1, pp. 147-165, 2002.

[9] Y. Q. Sun and M. Dhanasekar, "A dynamic model for the vertical interaction of the rail track and wagon system," International Journal of Solids and Structures, vol. 39, no. 5, pp. 1337-1359, 2002.

[10] Y. S. Lee, S. H. Kim, and J. Jung, “Three-dimensional finite element analysis model of high-speed train-track-bridge dynamic interactions," Advances in Structural Engineering, vol. 8, no. 5, pp. 513-528, 2005.

[11] F. T. K. Au, Y. S. Cheng, and Y. K. Cheung, "Effects of random road surface roughness and long-term deflection of prestressed concrete girder and cable-stayed bridges on impact due to moving vehicles," Computers and Structures, vol. 79, no. 8, pp. 853-872, 2001.

[12] F. T. K. Au, J. J. Wang, and Y. K. Cheung, "Impact study of cable-stayed railway bridges with random rail irregularities," Engineering Structures, vol. 24, no. 5, pp. 529-541, 2002.

[13] W. M. Zhai and C. B. Cai, "Train/track/bridge dynamic interactions: simulation and applications," Vehicle System Dynamics, vol. 37, supplement, pp. 653-665, 2003.

[14] W. M. Zhai, C. B. Cai, and K. Y. Wang, "Numerical simulation and field experiment of high-speed train-track-bridge system dynamics," Vehicle System Dynamics, vol. 41, pp. 677-686, 2004.

[15] Y. B. Yang, J. D. Yau, and Y. S. Wu, Vehicle-Bridge Interaction Dynamics: With Applications to High-Speed Railways, World Scientific, Singapore, 2004.

[16] H. Xia and N. Zhang, Dynamic Interaction of Vehicles and Structures, Science Press, Beijing, China, 2005 (Chinese).

[17] P. Lou, "Finite element analysis for train-track-bridge interaction system," Archive of Applied Mechanics, vol. 77, no. 10, pp. 707-728, 2007.

[18] M. Majka and M. Hartnett, "Dynamic response of bridges to moving trains: a study on effects of random track irregularities and bridge skewness," Computers \& Structures, vol. 87, no. 19-20, pp. 1233-1252, 2009.

[19] P. Lou, Z. Yu, and F. T. K. Au, "Rail-bridge coupling element of unequal lengths for analysing train-track-bridge interaction systems," Applied Mathematical Modelling. Simulation and Computation for Engineering and Environmental Systems, vol. 36, no. 4, pp. 1395-1414, 2012.

[20] J. H. Lin, Y. H. Zhang, F. Lu, and Y. H. Zhang, "Seismic spatial effects for long-span bridges, using the pseudo excitation 
method," Engineering Structures, vol. 26, no. 9, pp. 1207-1216, 2004.

[21] J. Lin, W. Zhang, and J. Li, "Structural responses to arbitrarily coherent stationary random excitations," Computers and Structures, vol. 50, no. 5, pp. 629-633, 1994.

[22] J. Lin, W. Zhang, and F. W. Williams, "Pseudo-excitation algorithm for nonstationary random seismic responses," Engineering Structures, vol. 16, no. 4, pp. 270-276, 1994.

[23] F. Lu, D. Kennedy, F. W. Williams, and J. H. Lin, "Symplectic analysis of vertical random vibration for coupled vehicle-track systems," Journal of Sound and Vibration, vol. 317, no. 1-2, pp. 236-249, 2008.

[24] F. Lu, J. H. Lin, D. Kennedy, and F. W. Williams, "An algorithm to study non-stationary random vibrations of vehicle-bridge systems," Computers \& Structures, vol. 87, no. 3-4, pp. 177-185, 2009.

[25] Z. C. Zhang, J. H. Lin, Y. H. Zhang, Y. Zhao, W. P. Howson, and F. W. Williams, "Non-stationary random vibration analysis for train-bridge systems subjected to horizontal earthquakes," Engineering Structures, vol. 32, no. 11, pp. 3571-3582, 2010.

[26] Y. B. Yang and Y. S. Wu, "A versatile element for analyzing vehicle-bridge interaction response," Engineering Structures, vol. 23, no. 5, pp. 452-467, 2001.

[27] P. Lou, "Finite-element formulae for calculating the sectional forces of a Bernoulli-Euler beam on continuously viscoelastic foundation subjected to concentrated moving loads," Shock and Vibration, vol. 15, no. 2, pp. 147-162, 2008.

[28] P. Lou and Q. Zeng, "Formulation of equations of motion of finite element form for vehicle-track-bridge interaction system with two types of vehicles model," International Journal for Numerical Methods in Engineering, vol. 62, no. 3, pp. 435-474, 2005.

[29] N. M. Newmark, "A method of computation for structural dynamics," ASCE Journal of Engineering Mechanics, vol. 85, no. 3, pp. 67-94, 1959.

[30] K. J. Bathe and E. L. Wilson, Numerical Methods in Finite Element Analysis, Prentice Hall, Englewood Cliffs, NJ, USA, 1976.

[31] W. M. Zhai, W. J. Han, C. B. Cai, and Q. C. Wang, "Dynamic properties of high-speed railway slab tracks," Journal of the China Railway Society, vol. 21, no. 6, pp. 65-69, 1999 (Chinese).

[32] Y.-S. Wu and Y.-B. Yang, "Steady-state response and riding comfort of trains moving over a series of simply supported bridges," Engineering Structures, vol. 25, no. 2, pp. 251-265, 2003.

[33] W. M. Zhai and H. Xia, Train-Track-Bridge Dynamic Interaction Theory and Engineering Application, Science Press, Beijing, China, 2011, (Chinese).

[34] V. K. Garg and R. V. Dukkipati, Dynamics of Railway Vehicle Systems, Academic Press, New York, NY, USA, 1984.

[35] G. Chen and W. M. Zhai, "Numerical simulation of the stochastic process of railway track irregularities," Journal of Southwest Jiaotong University, vol. 34, no. 2, pp. 138-142, 1999 (Chinese). 

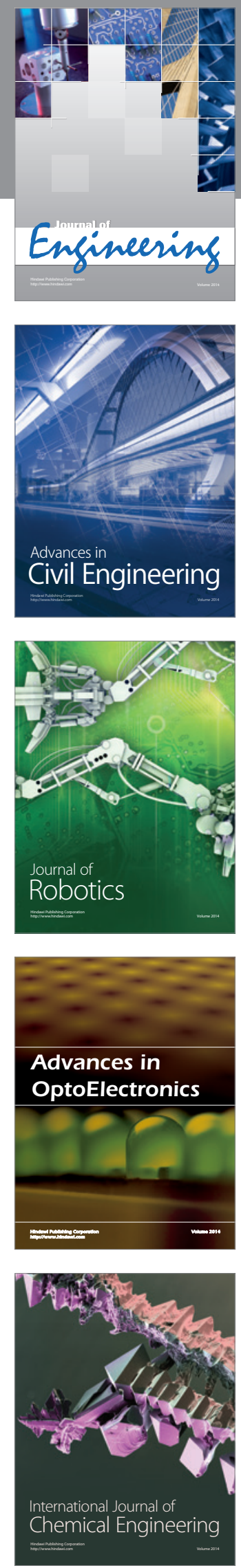

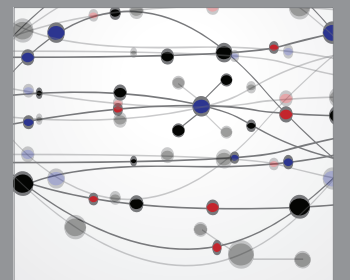

The Scientific World Journal
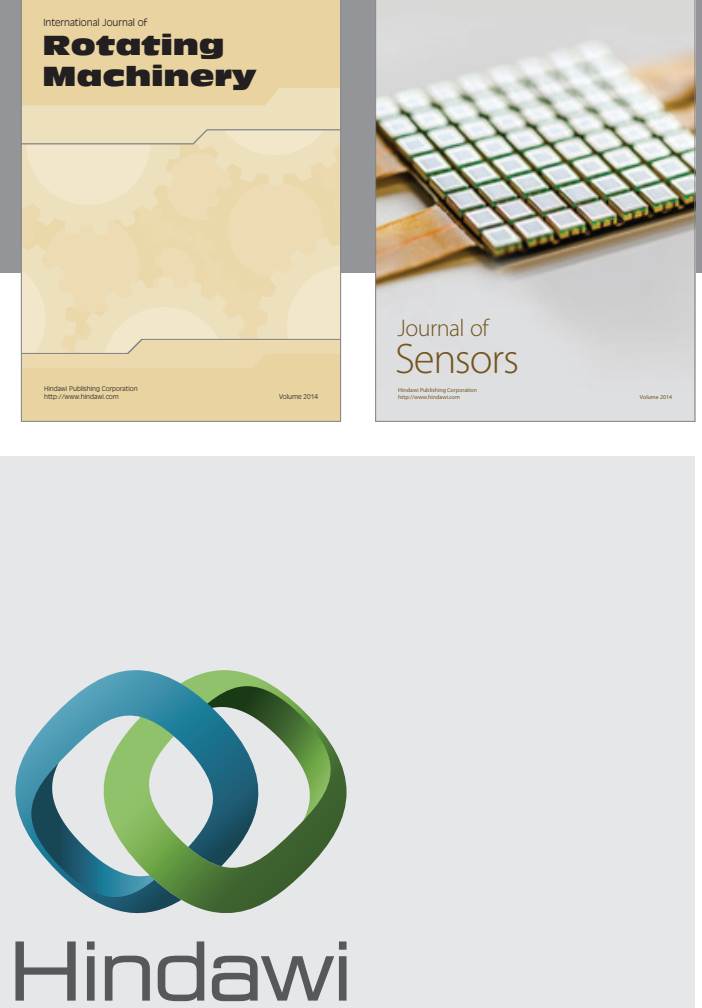

Submit your manuscripts at http://www.hindawi.com
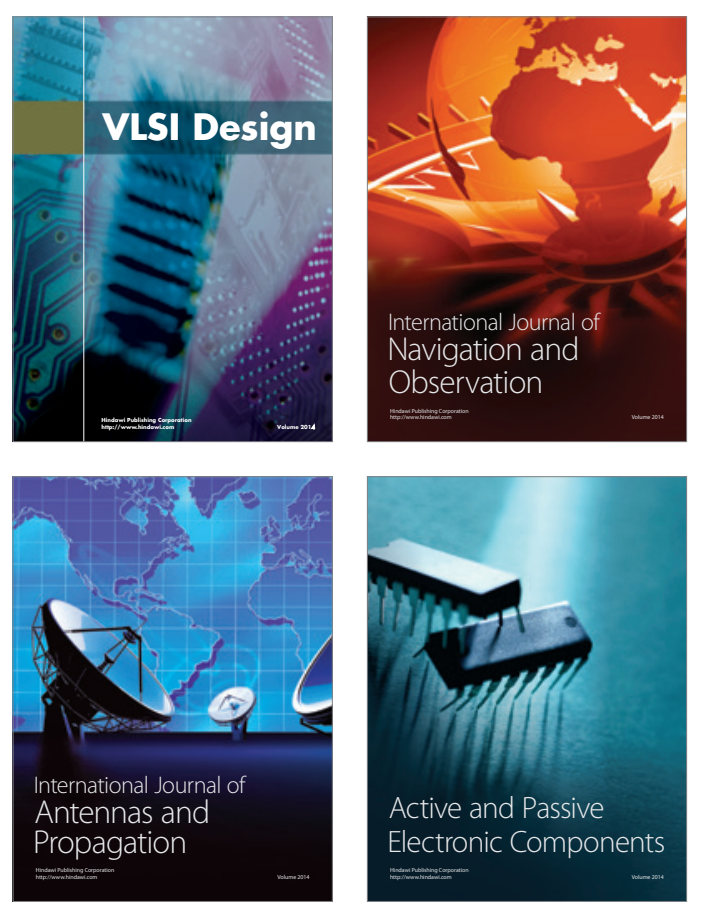
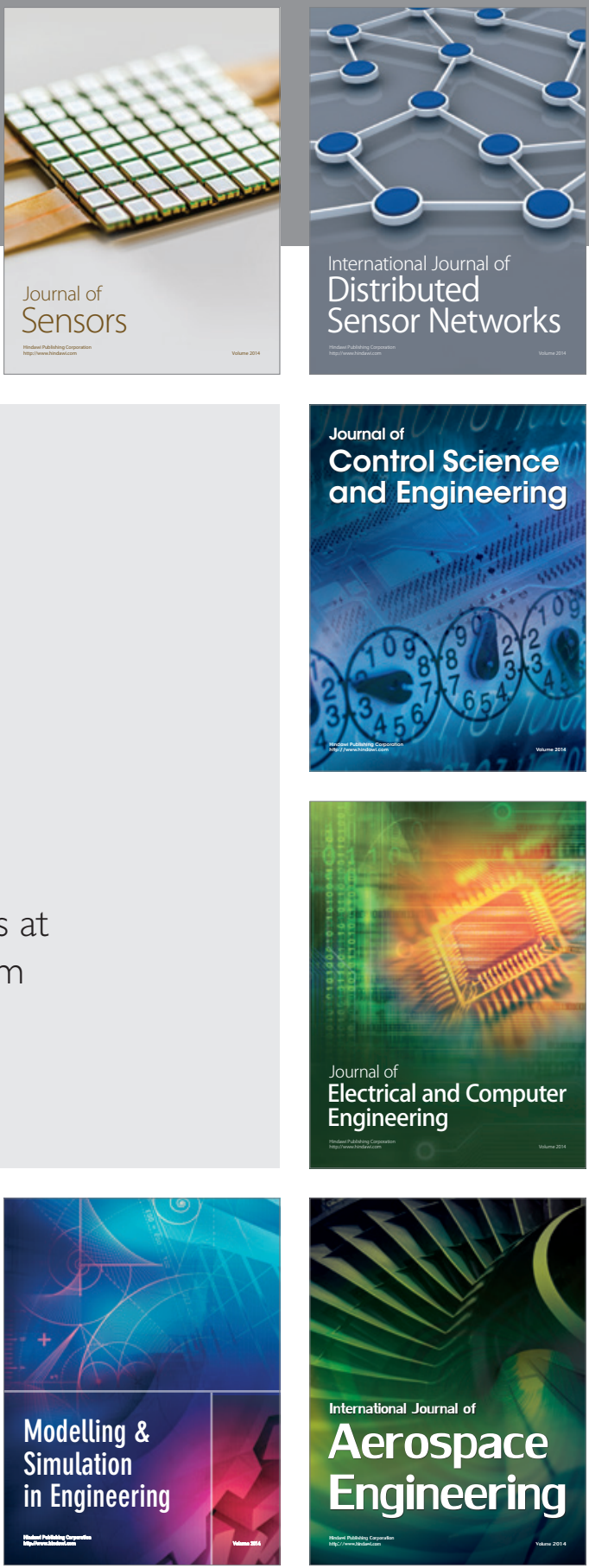

Journal of

Control Science

and Engineering
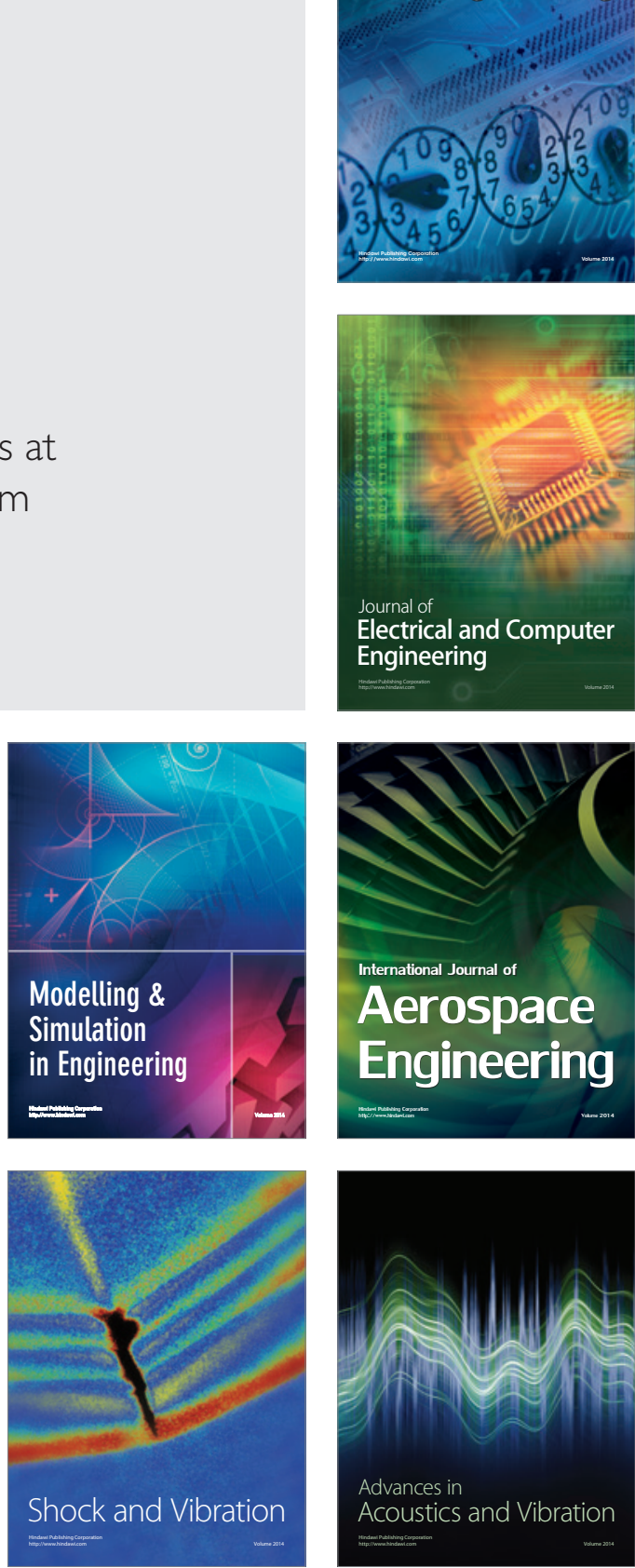